\title{
Pareto Optimal Allocation under Uncertain Preferences: Uncertainty Models, Algorithms, and Complexity
}

\author{
Haris Aziz \\ UNSW Sydney and Data61 CSIRO, Sydney, Australia \\ Péter Biró \\ Hungarian Academy of Sciences, and Corvinus University of Budapest, Hungary \\ Ronald de Haan \\ University of Amsterdam, the Netherlands \\ Baharak Rastegari \\ University of Southampton, Southampton, UK
}

\begin{abstract}
The assignment problem is one of the most well-studied settings in multi-agent resource allocation. Agents express preferences over indivisible items and then the items are allocated based on these preferences. Pareto optimality is regarded as a desirable property for the chosen allocation, requiring that no other allocation exists in which no agent is worse off and at least one agent is better of. We consider the assignment problem with the additional feature that agents' preferences involve uncertainty. The setting with uncertainty leads to a number of interesting questions including the following ones. How to compute an assignment with the highest probability of being Pareto optimal? What is the complexity of computing the probability that a given assignment is Pareto optimal? Does there exist an assignment that is Pareto optimal with probability one? We consider these problems under five natural uncertainty models. For all of the models, we present a number of algorithmic and complexity results highlighting the differences and similarities in the complexity of the models. We also present some general characterization and algorithmic results that apply to large classes of uncertainty models.
\end{abstract}

Keywords: Fair Division, Resource Allocation, Pareto optimality, Uncertain

Email addresses: haris.aziz@unsw.edu.au (Haris Aziz), biro.peter@krtk.mta.hu (Péter Biró), me@ronalddehaan.eu (Ronald de Haan), b.rastegari@soton.ac.uk (Baharak Rastegari) 
preferences

\section{Introduction}

Multi-agent resource allocation and dealing with uncertainty are two major topics in AI. In this paper we examine optimal allocation of resources under uncertain preferences.

When preferences of agents are aggregated to identify a desirable social outcome, Pareto optimality is a minimal requirement. Pareto optimality stipulates that there should not be another outcome that is at least as good for all agents and better for at least one agent. We take Pareto optimality as a central concern and consider a richer version of the classical assignment problem where the twist is that agents may express uncertainty in their preferences. The assignment problem is a fundamental setting in which $n$ agents express preferences over $n$ items and each agent is to be allocated one item. The setting is a classical one in discrete allocation. Its axiomatic and computational aspects have been well-studied [2, 3, 7, 11, 19, 29, 30]. Our motivation for studying assignment with uncertain preferences is that agents' preferences may not be completely known because of lack of information or communication. In some settings, eliciting preferences from agents may be costly, so a central planner may want to only obtain, and provide a recommendation based on, a subset of the complete orders $[26,25,15]$. Another possible motivation is that agents are in fact virtual or 'bidding' agents who are each representing a group of real agents and the virtual agent's probabilistic preferences simply represent the composition of preferences of the real agents it represents.

Our work is inspired by recent work where the stable marriage problem under uncertain preferences was considered (see, e.g., [6]). Uncertainty in preferences has already been studied in voting [20]. Similarly, in auction theory, it is standard to examine Bayesian settings in which there is a probability distribution over the types of the agents. Although computational aspects of Pareto optimal outcomes have been intensely studied in various settings such as assignment, matching, housing markets, and committee voting [3, 9, 10, 16, 21, 23, 27], there has not been much work on Pareto optimality under uncertain preferences. In the presence of uncertainty, the goal of computing a Pareto optimal outcome can be replaced by focusing on computing outcomes that have the highest probability of being Pareto optimal. We will abbreviate Pareto optimal as PO. If an assignment is Pareto optimal with probability one, we will call it certainly PO. When computing assignments with highest probability of being PO, we take a centralized view of multi-agent resource allocation. ${ }^{1}$

We consider the following uncertainty models. All of them have been considered in other contexts including voting or two-sided stable matching $[5,6,14,24]$. We will discuss the models and their motivation later in the introduction.

${ }^{1}$ There is also increasing focus on decentralized approaches to resource allocation (see e.g., Chevaleyre et al. [13].) 
- Lottery Model: For each agent, we are given a probability distribution over linear preferences over the items.

- Joint Probability Model: A probability distribution over linear preference profiles is specified where a preference profile specifies (deterministic) preferences of each agent over items.

- Compact Indifference Model: Each agent reports a single weak preference list that allows for ties. Each complete linear order extension of this weak order is assumed to be equally likely. ${ }^{2}$

- Pairwise Model: Each agent reports independent pairwise probabilities over pairs of items. If agent $i$ prefers item $o$ over item $o^{\prime}$ with probability $p$, then she prefers $o^{\prime}$ over $o$ with probability $1-p$.

- Ranking Model: Each agent reports probabilities for an item being in a rank, for each item and each rank. ${ }^{3}$ The input for each agent can be viewed as a bistochastic matrix - i.e., the sum of probabilities of items being in a given rank is 1, and the sum of probabilities of an item being in one of the $n$ ranks is 1 as well.

Example 1. Consider the following assignment problem in which agent 1 has uncertain preferences in the lottery model where with probability 0.5 she prefers a over $b$ over $c$ and with probability 0.5 she prefers $b$ over a over $c$.

$$
\begin{aligned}
& 1: \quad a, b, c \quad(0.5) \\
& b, a, c \quad(0.5) \\
& 2: \quad b, a, c \\
& 3 \text { : } c, b, a
\end{aligned}
$$

The same uncertain preferences can be also represented in the joint probability model with a probability distribution over two preference profiles where each profile gets probability 0.5 .

$$
\begin{aligned}
& \text { 1: } a, b, c \quad 1: b, a, c \\
& 2: b, a, c \quad 2: \quad b, a, c \\
& 3: c, b, a \quad 3: c, b, a
\end{aligned}
$$

Equivalently, agent 1's uncertain preferences can be represented in the compact indifference model by a weak order in which $a$ and $b$ are tied and they are both strictly more preferred by agent 1 over $c$. The same uncertain preferences

\footnotetext{
${ }^{2}$ The assignment problem is also known as the House Allocation problem. Compact indifference model can be viewed as the assignment problem with ties, or as it is widely known in the literature as the House Allocation problem with Ties (HRT) [23], where any preference list obtained by breaking ties arbitrarily is possible, and all possible preferences have the same likelihood of being realized.

${ }^{3}$ Under a linear preference ordering, the most preferred item is ranked 1st, the second most preferred item is ranked $2 \mathrm{nd}$, and so on, with the least preferred item being ranked $n$ th.
} 
can also be represented in the pairwise model whereby agent 1 has probability 1 of preferring a over $c$ and $b$ over $c$. Agent 1 prefers a over $b$ with probability 0.5 and prefers $b$ over a with probability 0.5 as well. Finally, agent 1's preferences can also be represented in the ranking model as follows.

1
$b$
$c$$\left(\begin{array}{ccc}0.5 & 0.5 & 3 \\ 0.5 & 0.5 & 0.0 \\ 0.0 & 0.0 & 1.0\end{array}\right)$

The most natural computational problems that we will consider are as follows.

- PO-Probability: what is the probability that a given assignment is PO?

- AssignmentWithHighestPO-Probability: compute an assignment with the highest probability of being PO.

We also consider problems that are simpler than PO-ProbabILITY:

- IsPO-ProbabilityNon-Zero: for a given assignment, is its probability of being $\mathrm{PO}$ non-zero?

- IsPO-ProbabilityOne: for a given assignment, is its probability of being $\mathrm{PO}$ one?

Additionally, we consider two problems connected to AssignmentWithHighestPO-ProbabiLity:

- ExistsPossiblyPO-Assignment: does there exist an assignment that is $\mathrm{PO}$ with non-zero probability?

- ExistsCertainlyPO-Assignment: does there exist an assignment that is $\mathrm{PO}$ with probability one?

Note that ExistsPossiblyPO-AssignMent is trivial for all uncertainty models in which the certainly preferred relation is acyclic. An agent certainly prefers an item to another if the preference is with probability 1 . The reason for the triviality is that the certainly preferred relation can be completed in a way so that it is transitive, and then for the completed deterministic preferences there exists at least one PO assignment. We call an uncertainty model reasonable if, for any subset of items $O^{\prime} \subseteq O$ and any agent $i \in N$, it can be checked in polynomial time whether $o \in O^{\prime}$ is a possibly most preferred item for $i$ among items in $O^{\prime}$. It is easy to verify that the lottery, joint probability, compact indifference, and the pairwise models are all reasonable. Although it is not obvious, we will show in Section 8 that the ranking model is reasonable as well.

We say that a given uncertainty model is independent if any uncertain preference profile $L$ under the model can be written as a product of uncertain preferences $L_{a}$ for all agents $a$, where all $L_{a}$ 's are independent [6]. Note that the joint probability model is not independent, but all the other problems that we study are independent. 


\begin{tabular}{ccccc}
\hline & Compact & Independent & Acylic & Reasonable \\
Uncertainty Model & & & & \\
\hline Lottery & $x$ & $\checkmark$ & $\checkmark$ & $\checkmark$ \\
Joint Probability & $x$ & $x$ & $\checkmark$ & $\checkmark$ \\
Compact Indifference & $\checkmark$ & $\checkmark$ & $\checkmark$ & $\checkmark$ \\
Pairwise & $\checkmark$ & $\checkmark$ & $x$ & $\checkmark$ \\
Ranking & $\checkmark$ & $\checkmark$ & $\checkmark$ & $\checkmark$ \\
\hline
\end{tabular}

Table 1: Properties of models. We say that a model is compact if its representation is polynomial in the number of agents and items. We say the model is acyclic if the certainly preferred relation of each agent is acyclic.

Contributions. We initiate the first study of computational aspects of Pareto optimal allocation under uncertain preferences. We present several general insights and results that apply to large classes of uncertainty models.

We present a comprehensive set of results for five uncertainty models that have not been considered in the context of Pareto optimal allocation. Our technical results are summarized in Table 2. Note that many of our interesting technical results are computational hardness results which therefore carry over to any settings in which an agent may find certain items unacceptable and/or an agent may be genuinely indifferent between two or more items.

Concretely, (1) We show that for all the models, ExistsCertainlyPOAssignment is NP-complete. It therefore follows that (2) AssignmentWithHighestPO-Probability is NP-hard for all the models. In view of these results, we see that as we move from deterministic preferences to uncertain preferences, the complexity of computing Pareto optimal assignments jumps significantly. (3) However, we show that for a general class of independent uncertainty models, both problems IsPO-ProBABILITYNON-ZERO and IsPO-Probability One can be solved in polynomial time. For all the uncertainty models that we consider, these problems are polynomial-time solvable. (4) Whereas PO-Probability is polynomial-time solvable for the joint probability model, we prove that the problem is \#P-complete for the other four models. (5) We additionally show that this problem becomes polynomial-time solvable for the lottery model if there is a constant number of uncertain agents. In fact, we show that the problem PO-ProBABILITY for the lottery model can be solved in fixed-parameter tractable time when parameterized by the number of uncertain agents.

Our algorithmic results for all the models except the ranking model also extend to the setting in which agents are allowed to declare some items unacceptable, as well as the setting in which there are unequal number of items and agents. However, extending the ranking model to capture either of these two generalizations is not straightforward. This is because we heavily use the assumption that the uncertainty matrix is bistochastic when defining various concepts for the ranking model. When we write that a problem can be solved in polynomial time for a certain setting, we mean polynomial in the input size which may be exponential in $n$. 


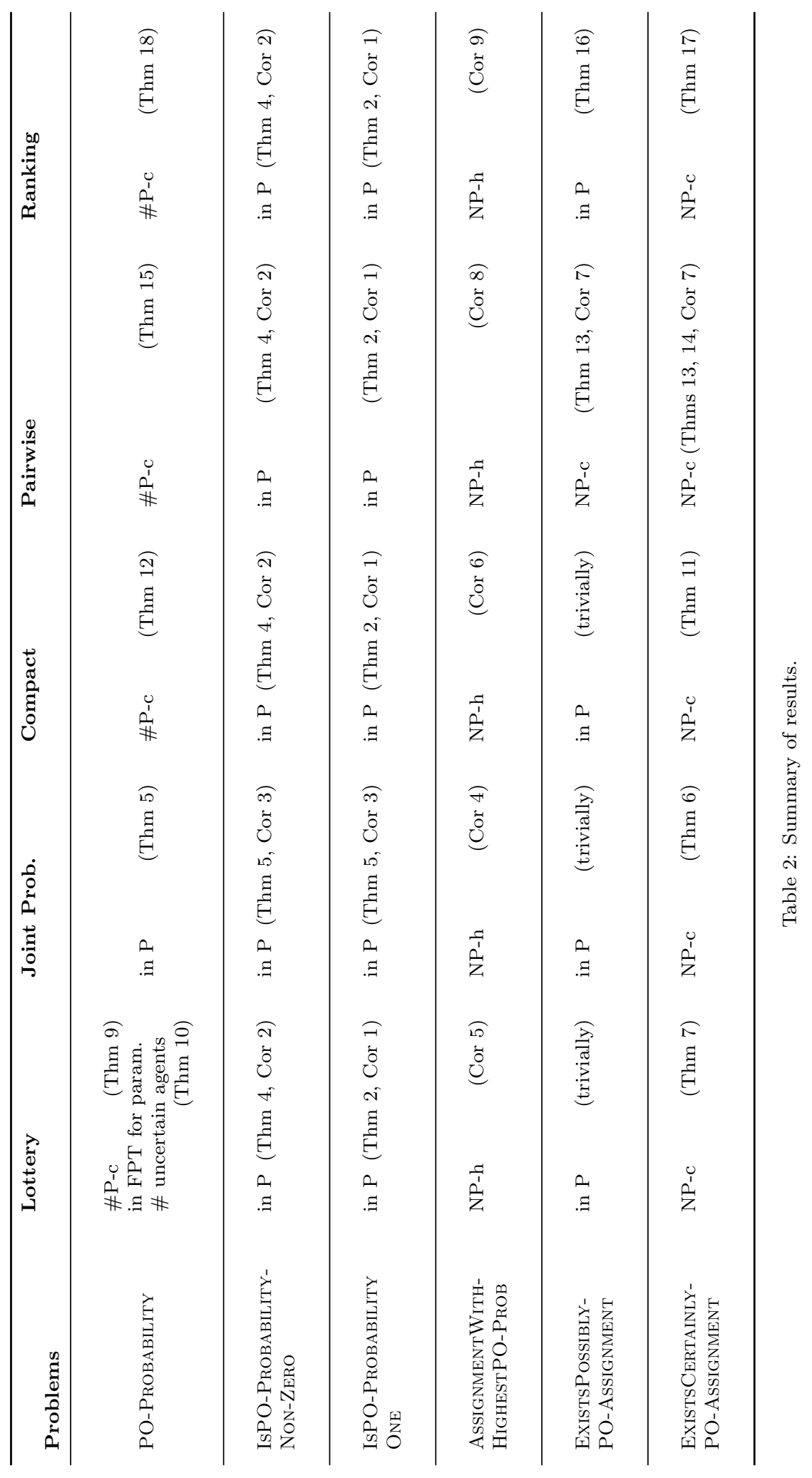


Justification of the Assignment Setting. In this paper we focus on a setting in which each agent has demand for exactly one item. Single-unit allocations are widely used in practice. In most scenarios, agents are seeking a single job, partner, apartment, day care, office desk, school seat, kidney etc.

We also assume that the number of items is equal to the number of agents and that no agent gets zero items. Our model, however, can also capture the setting in which the number of items is less than the number of agents and some agent may not get any item. Assigning zero items can be modelled by giving a null item to an agent.

Our hardness results carry over to the setting where an agent may be allocated several items. One issue when considering multi-unit allocations is that there are many ways of extending the preferences over items to preferences over bundles. Studying uncertainty in multi-unit allocations is an interesting topic for future work.

Discussion of the Uncertainty Models. The lottery and joint probability are two models that may not necessarily be polynomial in the number of agents or items. Note that the product of the independent uncertain preferences in the lottery model results in a probability distribution over preference profiles and hence can be represented in the joint probability model. However, the change in representation can result in an exponential blowup. Thus, whereas the joint probability model is more general than the lottery model, it is not as compact. In view of this, complexity results for one model do not directly carry over to results for the other model.

The compact indifference model explains scenarios where an agent may express indifference between some items because she does not have sufficient knowledge about their differences. The model is neutral to the relative ordering of these items and assumes that all linear orders consistent with the weak preferences are equiprobable. The model was considered by Aziz et al. [6] in the context of the two-sided marriage problem and with stability as the main concern. Although the model is quite restrictive, our hardness results for the model underline the fact that uncertainty even in restrictive models can lead to intractability.

The pairwise uncertainty model is well-studied in social sciences, in particular psychology, where people are asked to make repeated pairwise comparisons between different items or experiences. The model was formally studied in a related but different setting of the two-sided marriage problem [5], where the focus was on stability rather than Pareto optimality. The model is applicable to scenarios where the system has a record of similar pairwise choices and uses that record to find an outcome that has a high probability of being Pareto optimal.

The ranking model appeals to the idea that agents often ascribe ranks to items but they may not always be sure about the exact rank of each item (see, e.g., Dopazo and Martínez-Céspedes [14], Mazurek [24]). This notable approach to fuzzy rankings is applicable to scenarios where the system has a record of past ranking information and uses that fuzzy or aggregate record to find an outcome that has a high probability of being Pareto optimal. 
Any compact indifference preference profile can be represented by a lottery preference profile albeit with possibly an exponential blowup. In Section 8 we will discuss how the ranking model has connections with the lottery model.

In the lottery, joint probability, compact indifference and the ranking models, we assume that the underlying preferences of agents are linear orders. Hence the certainly preferred part of the relation is acyclic under these models.

In the pairwise probability model, we do not assume that comparisons are transitive and even allow agents to have cycles in their certainly preferred relations. Cyclic and intransitive preferences may happen in practice, e.g., when an agent is a virtual agent representing the preferences of a group of agents or a committee, or when agents' valuation functions over alternatives are complex and multidimensional. Table 1 summarizes the properties of the uncertainty models.

Roadmap. The remainder of the paper is structured as follows. We discuss some technical preliminaries in Section 2. Then, in Section 3, we present some results that apply to several of the uncertainty models. We then present and discuss, in separate sections, our results for the joint probability model (Section 4), the lottery model (Section 5), the compact indifference model (Section 6), the pairwise model (Section 7), and the ranking model (Section 8). We conclude in Section 9 .

\section{Preliminaries}

An instance of the (deterministic) assignment problem is a triple $(N, O, \succ)$ where $N$ is the set of $n$ agents $\{1, \ldots, n\}, O=\left\{o_{1}, \ldots, o_{n}\right\}$ is the set of items, and the preference profile $\succ=\left(\succ_{1}, \ldots, \succ_{n}\right)$ specifies complete and asymmetric preferences $\succ_{i}$ of each agent $i$ over $O$. Note that in the classical assignment problem, agents' preferences are also assumed to be transitive, hence resulting in linearly ordered preferences. Let $\mathscr{R}(O)$ denote the set of all complete and asymmetric relations over the set of items $O$. Let $\succ_{S}$ denote the preference profile of agents in the set $S \subset N$.

An assignment is an allocation of items to agents such that each agent is allocated a unique item. We will represent assignments by a permutation over $O$ so that an item in the $i$-th position in the permutation is given to agent $i$. For example, We refer by $a b c$ the assignment in which 1 gets $a, 2$ gets $b$ and 3 gets $c$. For a given assignment $\omega$, let $\omega(i)$ denote the item allocated to agent $i$.

An assignment $\omega$ is $P O$ (Pareto optimal) if there does not exist another assignment $\mu$ such that $\mu(i)=\omega(i)$ or $\mu(i) \succ_{i} \omega(i)$ for all agents $i$, and $\mu(j) \succ_{j}$ $\omega(j)$ for some agent $j$. If such an assignment $\mu$ exists, then we say that $\mu$ Pareto dominates $\omega$.

In this work, we allow agents to express uncertainty in their preferences and consider five uncertainty models. For each agent $i$ we define the certainly preferred relation $\succ_{i}^{\text {cert }}$. We write $b \succ_{i}^{\text {cert }} c$ if and only if agent $i$ prefers $b$ over $c$ with probability 1 . Checking $b \succ_{i}^{\text {cert }} c$ is straightforward in the lottery, joint probability, compact indifference, and pairwise models. We will show in 
Section 8 that for the ranking model it can be checked in polynomial time whether $b \succ_{i}^{\text {cert }} c$.

An agent $i$ possibly prefers an item $b$ to item $c$ if and only if $i$ prefers $b$ over $c$ with nonzero probability. Given an agent $i$, a subset of items $O^{\prime} \subseteq O$, and an item $a \in O^{\prime}$, we say that $a$ is a possibly most preferred item for $i$ among the items in $O^{\prime}$ if there is a deterministic preference compatible with the uncertain preferences in which $a$ is preferred over all other items in $O^{\prime}$. Checking whether $a$ is a possibly most preferred item in $O^{\prime}$ is straightforward in the lottery, joint probability, compact indifference, and pairwise models. We will show in Section 8 that this problem can be solved in polynomial time for the ranking model as well.

In some of our results, we will use ideas based on serial dictatorship that is an assignment algorithm for agents with deterministic preferences. Serial dictatorship takes a straightforward greedy approach that is specified with respect to a permutation $\pi$ over $N$. The algorithm takes each agent in turn, according to the permutation $\pi$, and allocates the most preferred item on their preference list to them that has not been allocated yet. We will denote by $S D(N, O, \succ, \pi)$ the outcome of applying serial dictatorship with respect to permutation of agents $\pi$ over assignment problem $(N, O, \succ)$. Serial dictatorship is presented more formally as Algorithm 1.

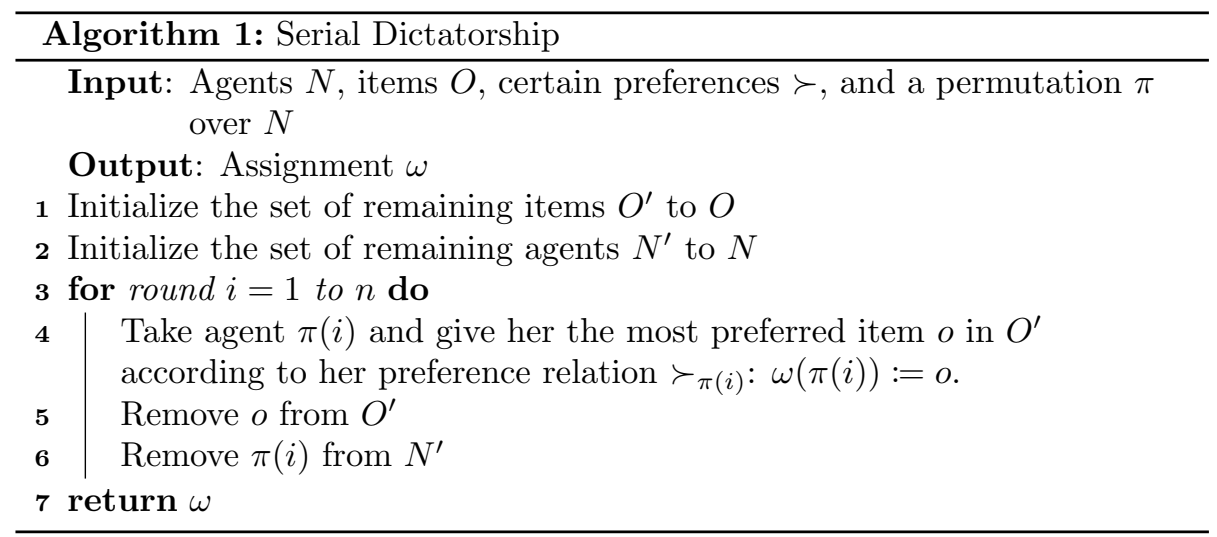

We will use the following characterization of Pareto optimal discrete assignments [1] that is defined with respect to outcomes of serial dictatorship.

Fact 1 (Abdulkadiroğlu and Sönmez [1]). Under deterministic strict preferences, an assignment is Pareto optimal if and only if it is an outcome of serial dictatorship.

Fact 1 also follows from Proposition 1 of Brams and King [12]. Next, we present another well-known characterization of $\mathrm{PO}$ assignments in deterministic settings. For any assignment $\omega$, the corresponding trading graph is a graph with vertex set $N \cup O$, where each item points to its owner and each agent points to items more preferred to her allocated item. The assignment $\omega$ admits a (trading) 
cycle $\left\langle o_{0}, i_{0}, o_{1}, i_{1}, \ldots, o_{k-1}, i_{k-1}, o_{0}\right\rangle$ if for all $j \in\{0, \ldots, k-1\}$ we have that $o_{j}=\omega\left(i_{j}\right)$ and $o_{j+1} \bmod k \succ_{i_{j}} o_{j}$. That is, a trading cycle is a cycle in the trading graph. The following fact is well-known.

Fact 2 (Abraham et al. [3]). For deterministic strict preferences, an assignment is PO if and only if its corresponding trading graph does not admit a cycle.

The trading cycle approach described for deterministic preferences can be extended in two different ways when considering uncertain preferences: (1) each agent can point to items that are certainly more preferred to her current item, or (2) each agent can point to items that are possibly more preferred to her current item. Note that computing the certainly preferred relation directly gives us the possibly preferred relation.

The facts above show that when preferences are deterministic, a Pareto optimal assignment can be computed and verified easily. In this paper we focus on finding and verifying Pareto optimal assignments in settings where agents have uncertain preferences.

Example 2. Consider the following assignment problem in which the uncertain preferences in the lottery model are as follows. The preferences are the same as in Example 1.

$$
\begin{aligned}
1: \quad a, b, c(0.5) & 2: \quad b, a, c \\
b, a, c(0.5) & 3: \quad c, b, a
\end{aligned}
$$

The same uncertain preferences can be also represented in the joint probability model with a probability distribution over two preference profiles.

Consider the assignment $\omega$ in which 1 gets $a, 2$ gets $b$, and 3 gets $c$. The probability of this assignment being $P O$ is 1 . This can be verified by considering each of the two possible preference profiles, and testing that no other assignment Pareto dominates $\omega$ under either of them. On the other hand, the assignment $\mu$ in which 1 gets $b, 2$ gets a, and 3 gets $c$ has 0.5 probability of being $P O$. This is because $\mu$ is not PO if the first possible preference list of agent 1 is realized, i.e., if $a \succ_{1} b \succ_{1} c$. To see this, notice that $\mu$ admits a trading cycle $\langle b, 1, a, 2, b\rangle$, implying that 1 and 2 prefer to trade their items and be both better off. If 1 and 2 trade their assigned items, we get assignment $\omega$. Assignment $\omega$ Pareto dominates assignment $\mu$ when the first possible preference list of agent 1 is realized.

Assignment $\mu$ is PO if the second possible preference list of agent 1 is realized, i.e., if $b \succ_{1} a \succ_{1} c$. Note that $\mu$ is the outcome of applying serial dictatorship with respect to permutation $\pi=1,2,3$ : (1) the first agent in the permutation, agent 1 , is allocated her most preferred item, item b, (2) agent 2 is allocated her most preferred available item, that is a, as b has been already allocated to 1, (3) agent 3 is allocated her most preferred available item, that is c.

\section{General Results}

In this section, we present some results that apply to several of the uncertainty models that we consider. 
We first present a couple of general results that apply to a large class of uncertainty models that satisfy independence. Recall that a given uncertainty model is independent if any uncertain preference profile $L$ under the model can be written as a product of uncertain preferences $L_{a}$ for all agents $a$, where all $L_{a}$ 's are independent.

Theorem 1. For any independent uncertainty model in which the certainly preferred relation can be computed in time $O(T)$, given an assignment $\omega$ it can be checked in time $O\left(T n^{2}\right)$ whether another assignment Pareto dominates $\omega$ with probability one.

Proof. Given an assignment $\omega$, we create a trading cycle graph $G^{c}$ in which each agent $i$ points to any item $o$ such that $o \succ_{i}^{\text {cert }} \omega(i)$. We then check whether $G^{c}$ admits a cycle or not. The construction of $G^{c}$ takes time $O\left(T n^{2}\right)$ and testing for the existence of a cycle takes time $O\left(n^{2}\right)$. We now claim that there exists a cycle in $G^{c}$ if and only if the assignment $\omega$ is Pareto optimal with probability zero.

If there exists a cycle in $G^{c}$, then another assignment Pareto dominates $\omega$ with probability one. The reason is that each agent prefers the item she points to over her assigned item with probability one. Hence, if we implement the trade in the cycle, each agent in the cycle gets a certainly more preferred item. Therefore, the new assignment Pareto dominates $\omega$ with probability one.

Now suppose that there is an assignment that Pareto dominates $\omega$ with probability one. Equivalently, there exists another assignment in which each agent with a different allocation gets a certainly more preferred item. But this means that there exists a cycle in $G^{c}$.

Theorem 2. For any independent uncertainty model in which the certainly preferred relation can be computed in polynomial time, IsPO-PROBABILITYONE can be solved in polynomial time. In particular, if the certainly preferred relation can be computed in time $O(T)$, IsPO-ProbabilityOne can be solved in time $O\left(T n^{2}\right)$.

Proof. We show that Algorithm 2 solves IsPO-ProbabiLityOne in polynomial time Given an assignment $\omega$, we create a trading cycle graph $G^{p}$ in which each agent $i$ points to any item $o$ such that $\omega(i) \nsucc_{i}^{\text {cert }} o$. The construction of $G^{p}$ can be done in time $O\left(n^{2}\right)$. We then check whether $G^{p}$ admits a cycle or not. This again can be done in time $O\left(T n^{2}\right)$. We claim that $\omega$ is Pareto optimal with probability one if and only if $G^{p}$ does not contain a cycle.

We first show that if there exists a cycle, then it is not the case that $\omega$ is $\mathrm{PO}$ with probability one. Existence of a cycle implies that each agent in the cycle prefers, with non-zero probability, another item to what she has received, which in turn implies that if we implement the cycle then each of these agents will receive an item that is more preferred with non-zero probability. Therefore $\omega$ is Pareto dominated with non-zero probability.

If it is not the case that $\omega$ is Pareto optimal with probability one, then it must be that another assignment Pareto dominates it with non-zero probability. Equivalently, there exists another assignment in which each agent with a 
different allocation gets a different item that is more preferred with non-zero probability. But this means that there exists a cycle in $G^{p}$.

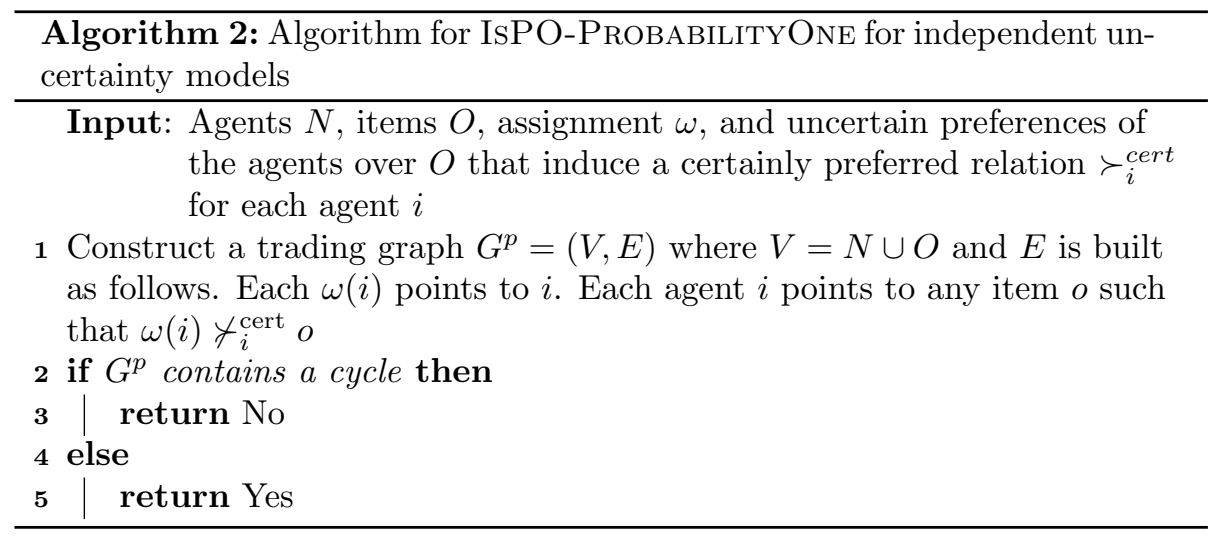

Since all the models we consider, except for the joint probability model, are independent, as a corollary, we get the following statement.

Corollary 1. For the lottery, compact indifference, pairwise, and the ranking uncertainty models, IsPO-PROBABILITYONE can be solved in polynomial time.

The proof of Theorem 2 provides the following characterization of certainly PO assignments under independent uncertainty models: an assignment is certainly PO if and only if the trading graph corresponding to "possibly more preferred' part of the relation does not admit a cycle. Analogously, one may presume that an assignment is possibly $\mathrm{PO}$ if and only if the trading graph $G^{c}$ (corresponding to the certainly preferred part of the relation) does not admit a cycle. However, it can be shown that the latter condition is necessary but not sufficient for possible Pareto optimality. See the following example.

Example 3. Agents 1 and 2 have two possible linear preferences, each with probability 0.5 , and agent 3 has one linear preferences. For agent 1 we have $a \succ_{1} b \succ_{1} c$ with probability 0.5 and $c \succ_{1} b \succ_{1}$ a with probability 0.5 . For agent 2 we have $b \succ_{2} a \succ_{2} c$ with probability 0.5 and $c \succ_{2} a \succ_{2} b$ with probability 0.5 . For agent 3 we have $b \succ_{3} c \succ_{3} a$. The preferences are summarized as follows.

$$
\begin{aligned}
& 1: \quad a, b, c \quad(0.5) \\
& c, b, a \quad(0.5) \\
& 3: \quad b, c, a \quad(1)
\end{aligned}
$$

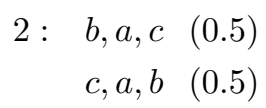

Let $\omega=$ bac and we ask whether $\omega$ is possibly PO. It is easy to see that the trading cycle graph $G^{c}$ (corresponding to the certainly preferred relations) 
does not admit a cycle, since only agent 3 strictly prefers another choice to her allocated item.

However, $\omega$ is not possibly $P O$ and this is easy to check by considering all cases. If the first preference list of agents 1 and 2 are realized, then assignment abc Pareto dominates $\omega$. If the first preference list of agent 1 and the second preference list of agent 2 are realized, then assignment acb Pareto dominates $\omega$. If the second preference list of agent 1 is realized, then assignment cab Pareto dominates $\omega$.

The following results are based on the notion of serial dictatorship (SD). In the literature, SD is defined for linearly ordered preferences. We noted earlier that under pairwise preferences, we allow agents to have cycles in their preferences; e.g., an agent may certainly prefer $b$ to $c, c$ to $d$, and $d$ to $b$. There is nothing to prevent one from running SD on deterministic pairwise preferences. However, when an agent's turn arrives she may not find an item she most prefers, due to the existence of a cycle, in which case SD must abort without any solution because the agent whose turn came, could not get her most preferred available item. It can thus be shown easily that Fact 1 applies to pairwise preferences as well. We present the following general theorem that applies to any uncertainty model and is a general characterization of possibly PO assignments.

Theorem 3. For any uncertainty model, an assignment $\omega$ is possibly $P O$ if and only if there is some permutation $\pi$ of agents and some preference profile that has non-zero probability under which when serial dictatorship is applied with respect to $\pi$, each agent in her turn gets item $\omega(i)$.

Proof. $(\Longleftarrow)$ Follows from Fact 1. If some deterministic preference profile $\succ^{\prime}$ has non-zero probability such that $\omega$ is the outcome of $S D\left(N, O, \succ^{\prime}, \pi\right)$, then $\omega$ is possibly $\mathrm{PO}$.

$(\Longrightarrow)$ Since $\omega$ is possibly PO, it is PO with respect to some realizable deterministic profile $\succ^{\prime}$. We show that $\omega=S D\left(N, O, \succ^{\prime}, \pi\right)$ for some permutation of $\pi$ agents. Take any partial assignment $\omega^{\prime} \subset \omega$ that allocates to a subset of agents $S \subset N$; that is, $\omega^{\prime}(i)=\omega(i)$ for all $i \in S$. Denote the items that are allocated in $\omega^{\prime}$ by $O^{\prime}$. (Note that $\omega^{\prime}$ and hence $S$ and $O^{\prime}$ can be empty sets.) We claim that there exists some agent $i \in N \backslash S$ such that, with respect to $\succ^{\prime}, \omega(i)$ is the most preferred item of agent $i$ among the set of items $O \backslash O^{\prime}$ (note that, by the definition of $\omega^{\prime}$ and $O^{\prime}, \omega(i)$ is in $\left.O \backslash O^{\prime}\right)$. Suppose for a contradiction that there exists no such an agent. Therefore, every agent $j \in N \backslash S$ is interested in, and in the corresponding trading graph points to, an item (or more) that is held (according to $\omega$ ) by another agent in $N \backslash S$. This implies the existence of a trading cycle where some agents in $N \backslash S$ can exchange items among themselves to get a more preferred item than in $\omega$, implying that $\omega$ is not PO with respect to the deterministic profile $\succ^{\prime}$, a contradiction. Therefore, we have established that starting with $\omega^{\prime}=\emptyset$, we can obtain $\omega$ by iteratively finding an agent who has not been allocated yet and, according to $\succ^{\prime}$, has $\omega(i)$ as her most preferred item among the unallocated ones. Such an agent always exists as proved above. Let the order in which the agents are chosen be $\pi$. It is easy to verify that $\omega=S D\left(N, O, \succ^{\prime}, \pi\right)$. 
We recall an uncertainty model reasonable if, for any subset of items $O^{\prime} \subseteq O$ and any agent $i \in N$, it can be checked in polynomial time whether $o \in O^{\prime}$ is a possibly most preferred item for $i$ among items in $O^{\prime}$. All of our models are reasonable and independent, except the joint probability model. Next, we present another general result that applies to this large class of uncertainty models.

Theorem 4. For any independent and reasonable uncertainty model, IsPOPROBABILITYNoN-ZERO can be solved in polynomial time. In particular, if it can checked in time $O(T)$ whether an item is a possibly most preferred item in a subset of items for a given agent, then ISPO-PROBABILITYNON-ZERO can be solved in time $O\left(T n^{2}\right)$.

Proof. Consider an assignment $\omega$ that we want check whether it is possibly PO. We use the following algorithm (presented as Algorithm 3) that builds a permutation $\pi$ of agents such that serial dictatorship produces $\omega$ given $\pi$, if and only if $\omega$ is possibly PO. To start with, we initialize the set of remaining items to $O$, the remaining agents to $N$, and the permutation of the agents $\pi$ to an empty list. We then repeat the following procedure until no more items are left, or the procedure returns no. Check if there exists some agent $i$ such that $\omega(i)$ is a possibly most preferred items for $i$ amongst the available items. If no such agent exists, return no. Otherwise, if such an $i$ exists, append $i$ to the permutation $\pi$, remove $i$ from the set of remaining agents, and remove $\omega(i)$ from the set of available items. This procedure halts in time $O\left(T n^{2}\right)$. Let $\succ_{i}^{\prime}$ denote a preference of agent $i$ that has $\omega(i)$ as the most preferred remaining item.

It is easy to verify that if the algorithm returns $\pi$, then $S D\left(N, O, \succ^{\prime}, \pi\right)=\omega$, and thus $\omega$ is possibly PO. We now show that if the algorithm returns no, then $\omega$ is $\mathrm{PO}$ with zero probability. Consider the first point in the algorithm where for no agent $i$ we have $\omega(i)$ as a possibly most preferred available item for $i$. This means that no remaining agent gets her most preferred item (for any possible deterministic preferences) among the available items. Therefore, for each realization of the preference profiles, each of the remaining agents is interested in, and points to, another item held by another agent among the remaining agents. This implies the existence of a trading cycle for each realization of the preference profiles, where some remaining agents can exchange items among themselves to get a more preferred item than in $\omega$. Thus $\omega$ is PO with probability zero.

We note that the theorem above gives us the following corollary.

Corollary 2. For the lottery, compact indifference, pairwise, and the ranking uncertainty models, IsPO-PROBABILITYNON-ZERO can be solved in polynomial time.

\section{Joint Probability Model}

In this section, we provide results that are specific to the joint probability model. We first observe that the PO-PROBABILITY problem can be solved easily 


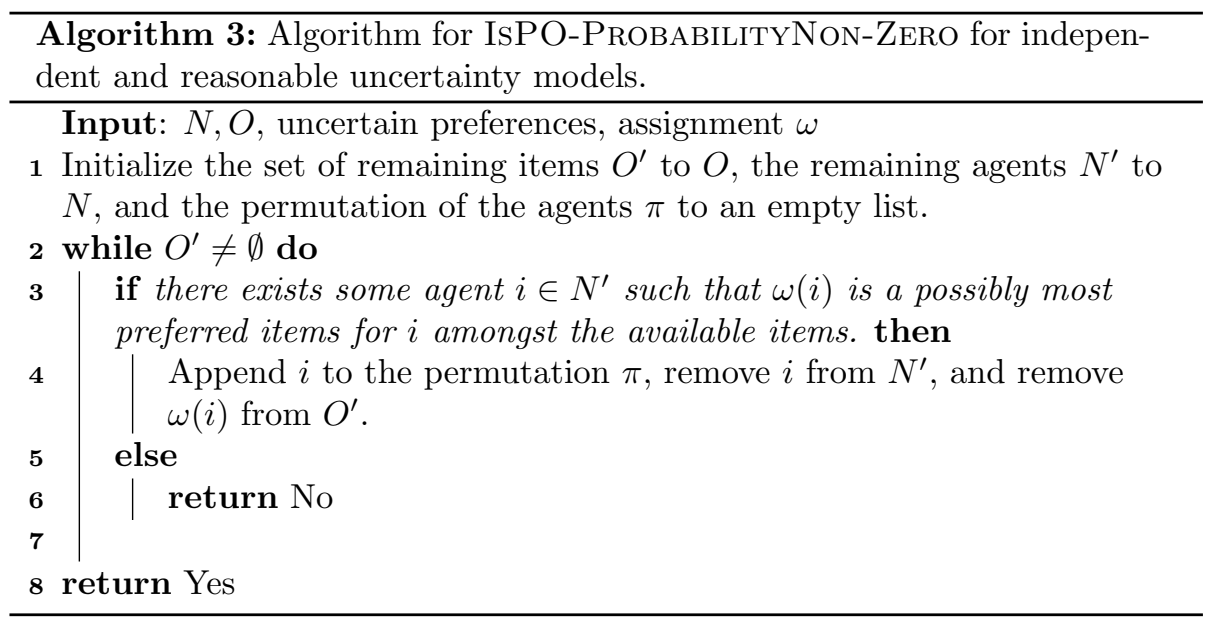

for the joint probability model.

Theorem 5. For the joint probability model, PO-ProbabiLity can be solved in polynomial time.

Proof. The probability that a given assignment is $\mathrm{PO}$ is equivalent to the probability weight of the preference profiles for which the assignment is PO. This can be checked as follows. We check the preference profiles for which the given assignment is PO (for one profile, this can be checked in linear time). Then we add the probabilities of those profiles for which the assignment is PO. The sum of the probabilities is the probability that the assignment is PO.

This gives us the following corollary.

Corollary 3. For the joint probability model, IsPO-ProbabilityNon-Zero and IsPO-PROBABILITYONE can be solved in polynomial time.

What about ExistsCertainlyPO-Assignment? This problem is equivalent to checking whether the sets of PO assignments under all possible realizations of preference profiles have a non-empty intersection. We show that this problem is NP-complete even when the probability distribution is over two linear preference profiles.

We reduce from the NP-complete problem SERIALDictatorshipFEAsibiLity. The question for this problem is to check whether there exists a permutation of agents for which serial dictatorship allocates a given item $o$ to a given agent $i$ [28].

SERIALDICTATORSHIPFEASIBILITY

Input: $\quad(N, O, \succ, i \in N, o \in O)$

Question: Does there exist a permutation of agents for which serial dictatorship gives item $o$ to agent $i$ ? 
It follows from Fact 1 that the following problem is also NP-complete: check whether there exists a Pareto optimal assignment in which a specified agent $i$ gets a specified item $o$.

Theorem 6. For the joint probability model, ExistsCertainlyPOAssignment is NP-complete even when the probability distribution is over two linear preference profiles.

Proof. The problem ExistsCertainlyPO-Assignment is in NP because it can be checked in polynomial time whether a given assignment is certainly PO or not (Corollary 3).

To prove NP-hardness, we reduce from the NP-complete problem SERIALDictatorshipFeasibility. Let $(N, O, \succ, i \in N, o \in O)$ be an instance of this problem. We construct a joint probability over two preference profiles. One of the profiles is the same as $\succ$. In the other preference profile $\succ^{\prime}$, agent $i$ has $o$ as the most preferred item and has the same order of preference over all other items as in $\succ_{i}$. Each agent $j \in N \backslash\{i\}$ has $o$ as the least preferred item. As for the other items, each $j \in N \backslash\{i\}$ has the same preferences over the items in $O \backslash\{o\}$ as in $\succ_{j}$.

Our first observation is that if assignment is PO under profile $\succ^{\prime}$ then $i$ gets $o$ in it.

Claim 1. If an assignment is $P O$ under profile $\succ^{\prime}$, then $i$ gets $o$ in it.

Proof. The argument is as follows. If $i$ does not get $o$, then an agent $j \neq i$ gets it. However both $i$ and $j$ get a more preferred item under profile $\succ^{\prime}$ by exchanging their items.

We now prove that we have a yes instance of SERIALDiCTATORSHIPFEASIBILITY if and only if there exists a certainly PO assignment.

Assume that there exists a certainly PO assignment. Then, it must be PO under $\succ^{\prime}$ implying that, by our claim above, $i$ gets $o$ in this assignment. The same assignment must also be PO under profile $\succ$ which implies that there exists an assignment that is $\mathrm{PO}$ under profile $\succ$ in which $i$ gets $o$. In light of Fact 1, this implies that there exists a serial dictatorship the outcome of which under profile $\succ$ is the same assignment. Hence, we have a yes instance of SERIALDiCTATORSHIPFEASIBILITY.

Now consider the case when we have a yes instance of SerialDictatorSHIPFEASIBILITY. This means that there is a permutation of agents $\pi$ under which $i$ gets $O$ when serial dictatorship is run. Let us call this assignment by $\omega$. We want to prove that $\omega$ is PO under each possible preference profile. Due to Fact 1, $\omega$ is PO under preference profile $\succ$. So it remains to show that it is PO under $\succ^{\prime}$. Again due to Fact 1, it is sufficient to prove that for profile $\succ^{\prime}$, there exists a corresponding permutation of agents under which the outcome of serial dictatorship is $\omega$. In fact, we show that $S D\left(N, O, \succ^{\prime}, \pi\right)=\omega$-i.e., the outcome of applying serial dictatorship with permutation of agents $\pi$ is $\omega$ even 
if the preference profile is $\succ^{\prime}$ instead of $\succ$. In order to prove the statement we prove the following claim where we track the set of remaining items as specified in Algorithm 1.

Claim 2. The following are the same at each round, when applying serial dictatorship (Algorithm 1) to profiles $\succ$ and $\succ^{\prime}$, in both cases with respect to permutation $\pi$.

- the order in which items are allocated,

- the allocation of each agent, and

- set of remaining items.

Proof. The claim can be proved via induction on the number of rounds of serial dictatorship that have already taken place. For the base case, let us consider agent $\pi(1)$. If $\pi(1)=i$, then $\pi(1)$ picks $o$ under both preference profiles. This is because, by construction (1) $\pi$ is a permutation of agents under which $i$ gets $o$ when serial dictatorship is applied on $\succ$, and (2) $i$ has $o$ as her most preferred item under $\succ^{\prime}$. If $\pi(1) \neq i$, then $\pi(1)$ picks some item $o^{\prime} \neq o$ in $\omega=S D(N, O, \succ, \pi)$. Note that for $\pi(1)$, her most preferred item in both profiles must be $o^{\prime}$. Hence by the end of the first round, the same item has been given to the same agent in both $\succ$ and $\succ^{\prime}$.

For the induction, let us assume that $k$ rounds have taken place and the order in which items are allocated, the allocation of each agent in the first $k$ rounds and the set of unallocated items $T$ is the same under both profiles $\succ$ and $\succ^{\prime}$. Now consider agent $\pi(k+1)$. If $\pi(k+1)=i$, then $i$ picks item $o$ under $\succ$, implying that $o \in T$, which in turn implies that $i$ must pick $o$ under $\succ^{\prime}$ since $o$ is her most preferred item in $O$ under preference $\succ_{i}^{\prime}$ and hence her most preferred item in $T$. It remains to show what happens when $\pi(k+1) \neq i$. In that case $\pi(k+1)$ picks some item $o^{\prime} \neq o$ in $\omega=S D(N, O, \succ, \pi)$. This means that $o^{\prime}$ is the most preferred item of agent $\pi(k+1)$ in set $T \subset O$ under preference profile $\succ$, implying that $o^{\prime}$ is the most preferred item of agent $\pi(k+1)$ in set $T$ under preference profile $\succ^{\prime}$ as well. This completes the proof of the claim.

We have thus proved that the outcome of applying serial dictatorship with respect to permutation of agents $\pi$ is $\omega$ under both preference profiles $\succ$ and $\succ^{\prime}$. Thus $\omega$ is PO under both possibly realizable preference profiles. To conclude, we have proved that there exists a certainly PO assignment if and only if we have a yes instance of SerialDictatorshipFeasibility. Since SerialDicTATORSHIPFEASIBILITY is NP-complete, it follows that ExistsCERTAINLYPOAssignment is NP-complete.

Theorem 6 gives us the following corollary.

Corollary 4. For the joint probability model, AssignmentWithHighestPOPROB is NP-hard. 
Proof. Assume to the contrary that there exists a polynomial-time algorithm to solve AssignmentWithHighestPO-Prob. In that case, we can compute such an assignment $\omega$. By Corollary 3, it can be checked in polynomial time whether $\omega$ is PO with probability one or not. If $\omega$ is $\mathrm{PO}$ with probability one, then we know that we have a yes instance of ExistsCertainlyPO-Assignment. Otherwise, we have a no instance of ExistsCertainlyPO-Assignment. Hence EXIstsCERTAINLYPO-AssignMENT is polynomial-time solvable, a contradiction.

\section{Lottery Model}

In this section, we obtain some further results for the lottery model, that go beyond the results of Corollaries 1 and 2 .

First, we prove that the problem of checking whether there exists an assignment that is $\mathrm{PO}$ with probability one is NP-complete. Although the proof is similar to the proof of Theorem 6, we give a complete argument since a complexity result for the joint probability model does not directly imply a similar result for the lottery model.

Theorem 7. For the lottery model, Exists CertainlyPO-Assignment is NPcomplete even when each agent has at most two possible preferences.

Proof. The problem ExistsCertainlyPO-Assignment is in NP because it can be checked in polynomial time whether a given assignment is certainly PO or not (Corollary 1). To prove NP-hardness, we use an argument similar to that used in the proof of Theorem 6 .

We reduce from the NP-complete problem SerialDictatorshipFEASIBILITY. Let $(N, O, \succ, i \in N, o \in O)$ be an instance of this problem. We construct an instance of the lottery model in which each agent $j \in N$ has two preference lists where one of them is $\succ_{j}$. For agent $i$, we add another preference list $\succ_{i}^{\prime}$ in which $i$ 's most preferred item is $o$ and the rest of the items are in the same order as in $\succ_{i}$. For each other agent $j \in N \backslash\{i\}$, we add a preference list $\succ_{j}^{\prime}$ which is identical to $\succ_{j}$ except that $o$ is moved to the end of the list.

Our first observation is that an assignment is PO under profile $\succ^{\prime}$ only if $i$ gets $o$ in it. If $i$ does not get $o$, and agent $j \neq i$ gets it, then both $i$ and $j$ get a more preferred item under profile $\succ^{\prime}$ by exchanging their items. Hence if there is any assignment that is certainly PO then it must give $o$ to $i$.

We prove that there exists a certainly PO assignment if and only if we have a yes instance of SERIALDiCTATORSHIPFEASIBILITY.

If we have a no instance of SERIALDiCTATORSHIPFEASIBILITY, then it follows Fact 1 that in no assignment that is $\mathrm{PO}$ under $\succ$ agent $i$ gets $o$. On the other hand, an assignment is $\mathrm{PO}$ under $\succ^{\prime}$ only if $i$ receives $o$. Therefore, there does not exist any certainly $\mathrm{PO}$ assignment.

Now consider the case when we have a yes instance of SerialDictatorSHIPFEASIBILITY. This means that there is a permutation of agents $\pi$ under 
which $i$ gets $o$ when serial dictatorship is run. Let us call this assignment $\omega$. Due to Fact 1, $\omega$ is PO under preference profile $\succ$. We want to prove that $\omega$ is PO under each possible preference profile. Due to Fact 1, it is sufficient to prove that each possible realizable preference profile, there exists a corresponding permutation of agents under which the outcome of serial dictatorship is $\omega$.

In fact, we show that for each possible preference profile $\succ^{\prime \prime}$, $S D\left(N, O, \succ^{\prime \prime}, \pi\right)=\omega$ i.e., the outcome of applying serial dictatorship with respect to permutation of agents $\pi$ is $\omega$. In order to prove the statement we prove the following claim. (Recall that $\omega$ is $\mathrm{PO}$ under $\succ$ with respect to $\pi$.)

Claim 3. The following are the same at each round, when applying serial dictatorship to $\succ$ and any of the realizable preference profiles $\succ^{\prime \prime}$, in both cases with respect to permutation $\pi$

- the order in which items are allocated,

- the allocation of each agent, and

- set of remaining items.

Proof. The claim can be proved via induction on the number of rounds of serial dictatorship that have already taken place. For the base case, let us consider agent $\pi(1)$. If $\pi(1)=i$, then $\pi(1)$ picks $o$ in all her possible preferences. This is because, by construction (1) $\pi$ is a permutation of agents under which $i$ gets $o$ when serial dictatorship is applied on $\succ$, so it must be that $i$ ranks $o$ at the top of her list under $\succ_{i}$ and (2) $i$ has $o$ as her most preferred item under $\succ_{i}^{\prime}$ by construction. If $\pi(1) \neq i$, then $\pi(1)$ picks some item $o^{\prime} \neq o$ in $\omega=S D(N, O, \succ, \pi)$. Note that for $\pi(1)$, her most preferred item is the same in all possible profiles. Hence by the end of the first round, the same item has been given to the same agent in all the realizable preferences.

For the induction, let us assume that $k$ rounds have taken place and the order in which items are allocated, the allocation of each agent in the first $k$ rounds and the set of unallocated items $T$ is the same all the realizable preferences. Now consider the agent $\pi(k+1)$. If $\pi(k+1)=i$, then $i$ picks item $o$ under $\succ$, implying that $o \in T$, which in turn implies that $i$ must pick $o$ under $\succ_{i}^{\prime}$ since $o$ is her most preferred item in $O$ under $\succ_{i}^{\prime}$ and hence her most preferred item in $T$. It remains to show what happens when $\pi(k+1) \neq i$. In that case $\pi(k+1)$ picks some item $o^{\prime} \neq o$ in $\omega=S D(N, O, \succ, \pi)$. This means that $o^{\prime}$ is the most preferred item of agent $\pi(k+1)$ in set $T \subset O$ under preference list $\succ_{\pi(k+1)}$, implying that $o^{\prime}$ is the most preferred item of agent $\pi(k+1)$ in set $T$ under preference list $\succ_{\pi(k+1)}^{\prime}$ as well. This completes the proof of the claim.

We have thus proved that the outcome of applying serial dictatorship with respect to permutation $\pi$ is $\omega$ under all possible preference profiles. Thus $\omega$ is PO under each possibly realizable preference profile when we have a yes instance of SerialDictatorshipFeasibility. To conclude, we have proved that there 
exists a certainly $\mathrm{PO}$ assignment if and only if we have a yes instance of SERialDictatorshipFeasibility. Since SerialDictatorshipFeasibility is NP-complete and ExistsCertainlyPO-Assignment is in NP, it follows that ExistsCertainlyPO-Assignment is NP-complete.

Theorem 7 gives us the following corollary.

Corollary 5. For the lottery model, AssignmentWithHighestPO-Prob is NP-hard.

Proof. Assume to the contrary that there exists a polynomial-time algorithm to solve AssignmentWithHighestPO-Prob. In that case, we can compute such an assignment $\omega$. By Corollary 1, it can be checked in polynomial time whether $\omega$ is $\mathrm{PO}$ with probability one or not. If $\omega$ is $\mathrm{PO}$ with probability one, then we know that we have a yes instance of ExistsCertainlyPO-Assignment. Otherwise, we have a no instance of ExistsCertainlyPO-Assignment. Hence EXISTSCERTAinlyPO-AsSignMent is polynomial-time solvable, a contradiction.

In light of Corollaries 1 and 2, we know that for the lottery model it can be checked in polynomial time whether the PO probability of a given assignment is zero or one, respectively. We now turn to the problem of computing the probability that a given assignment is $\mathrm{PO}$. We first present a polynomial-time solution for a restricted setting, and then show that PO-ProbABILITY is \#Pcomplete for the lottery model in general.

Theorem 8. For the lottery model, if the number of uncertain agents in constant, then PO-ProBABILITY is polynomial-time solvable.

Proof. Let $\omega$ be a given assignment. Let constant $k$ denote the number of uncertain agents, and let the maximum number of preferences for any uncertain agent be $\ell$. Therefore, the maximum number of preference profiles that are realizable is $\ell^{k}$ which is still polynomial in the input since $k=O(1)$. For each possible preference profile $\succ$, it is easy to compute the probability of $\succ$ by simply computing the product of the probabilities of the preferences chosen for the uncertain agents. Hence, we have reduced the problem to the problem POProbability for the joint probability model which can be solved in polynomial time (Theorem 5).

Theorem 9. For the lottery model, PO-Probability is \#P-complete, even when restricted to the case where each agent has at most two possible preferences.

Proof. We show \#P-hardness by reduction from the \#P-complete problem Monotone-\#2SAT - count the number of satisfying assignments for a $2 \mathrm{CNF}$ formula that contains no negation [31].

MONOTONE-\#2SAT

Input: A 2CNF formula that contains no negation.

Question: Count the number of satisfying assignments. 
Let $\varphi$ be a monotone $2 \mathrm{CNF}$ formula with clauses $c_{1}, \ldots, c_{m}$ and variables $x_{1}, \ldots, x_{n}$. We construct an instance of PO-Probability as follows. Consider agents $1, \ldots, n$ and items $o_{1}, \ldots, o_{n}$, and take the assignment $\sigma$ that gives each agent i item $o_{i}$.

We construct the preferences of the agents as follows. Take an arbitrary agent $i$. Consider the set $\left\{j_{1}, \ldots, j_{u}\right\}$ of indices $j$ such that the clause $\left(x_{i} \vee x_{j}\right)$ occurs in $\varphi$-i.e., $\left\{j_{1}, \ldots, j_{u}\right\}=\left\{j \mid\left(x_{i} \vee x_{j}\right)\right.$ appears in $\left.\varphi\right\}$. (Without loss of generality, this set $\left\{j_{1}, \ldots, j_{u}\right\}$ is non-empty.) Suppose that $j_{1}<j_{2}<\cdots<j_{u}$, in order to fix an (arbitrary) order over these indices. With probability $\frac{1}{2}$, agent $i$ has $o_{i}$ at the top of her preference list, followed by the rest of the items in arbitrary order. With probability $\frac{1}{2}$, agent $i$ has the following preference: $o_{j_{1}} \succ_{i} \cdots \succ_{i} o_{j_{u}} \succ_{i} o_{i} \succ_{i} \cdots$, where the remaining items appear in arbitrary order after $o_{i}$.

This way, the possible preference profiles correspond one-to-one to the possible truth assignments over $x_{1}, \ldots, x_{n}$. Namely, taking the preference $o_{i} \succ_{i} \ldots$ for agent $i$ corresponds to setting $x_{i}$ to 1 , and taking the other preference for agent $i$ corresponds to setting $x_{i}$ to 0 . Moreover, each possible preference profile occurs with probability $\frac{1}{2^{n}}$.

We show that the number of satisfying assignments for $\varphi$ is equal to the number of preference profiles under which $\sigma$ is Pareto optimal. In particular, we show that $\sigma$ is $\mathrm{PO}$ under a preference profile if and only if the corresponding truth assignment $\mathrm{T}$ satisfies $\varphi$.

( $\Longrightarrow$ ) Take a possible preference profile $\succ$ under which $\sigma$ is PO and suppose, for a contradiction, that the corresponding truth assignment $\mathrm{T}$ does not satisfy $\varphi$. That is, there is some clause $c=\left(x_{i} \vee x_{j}\right)$ that is not satisfied, implying that in $\mathrm{T}$ both $x_{i}$ and $x_{j}$ are set to 0 . Then we know that agent $i$ prefers $o_{j}$ to $o_{i}$ and agent $j$ prefers $o_{i}$ to $o_{j}$, hence they are willing to swap their assigned items. Therefore $\sigma$ is not Pareto optimal under $\succ$, a contradiction.

$(\Longleftarrow)$ Take a possible preference profile $\succ$ and suppose that the corresponding truth assignment $\mathrm{T}$ satisfies $\varphi$. We show that we cannot find a Pareto improvement of $\sigma$, implying that $\sigma$ is PO. Take an arbitrary agent $i$. First suppose that T sets $x_{i}$ to 1 . This means that agent $i$ prefers $o_{i}$ to all other items, and so she is not willing to exchange it with another item. Now, suppose that $\mathrm{T}$ sets $x_{i}$ to 0 . Take the set $\left\{j_{1}, \ldots, j_{u}\right\}$ of indices such that the clause $\left(x_{i} \vee x_{j}\right)$ occurs in $\varphi$. As $x_{i}$ is set to 0 , this means that $i$ prefers $o_{j_{1}}, \ldots, o_{j_{u}}$ to $o_{i}$ and is willing to exchange $o_{i}$ with either of these items (but no other item). Because $\mathrm{T}$ satisfies $\varphi$, we know that $\mathrm{T}$ sets $x_{j_{1}}, \ldots, x_{j_{u}}$ to 1 , and consequently, agents $j_{1}, \ldots, j_{u}$ prefer items $o_{j_{1}}, \ldots, o_{j_{u}}$ over all other items (respectively). So neither of these agents is willing to exchange their assigned item with $o_{i}$. Therefore, as no Pareto improvement exists, $\sigma$ is Pareto optimal.

The number of satisfying truth assignments of $\varphi$ is then exactly equal to $2^{n}$ times the probability that assignment $\sigma$ is Pareto optimal. Thus, POProbability is \#P-hard, even when restricted to the case where each agent has at most two possible preferences.

Next, we argue that PO-Probability is in \#P. Technically speaking, the class \#P consists of counting problems, which are functions $f: \Sigma^{*} \rightarrow \mathbb{N}$. We 
can consider PO-ProBABILITY as such a function producing natural numbers in the following way. Without loss of generality, suppose that the probabilities in the input are all given as rational numbers with the same denominator $d$. (We can transform the input in polynomial time to an equivalent input that satisfies this property.) Then the probability that the given assignment is Pareto optimal is $\frac{z}{d^{n}}$ for some positive integer $z$. We then consider the problem POPROBABILITY as the function that returns $z$, rather than the rational $\frac{z}{d^{n}}$.

We argue membership in \#P by describing a nondeterministic Turing machine $\mathbb{M}$ that has the property that for each input, the number of accepting paths of $\mathbb{M}$ for this input equals the number $z$ that corresponds to the probability that the given matching is Pareto optimal. The existence of such a Turing machine implies membership in \#P [31]. The machine $\mathbb{M}$ operates as follows. For each agent $a_{i}$, it uses nondeterminism to generate $d$ different (partial) computation paths. These partial computation paths are concatenated, resulting in $d^{n}$ total computation paths. Suppose that the input specifies $\ell$ possible preference orders for agent $a_{i}$, occurring with probabilities $\frac{u_{1}}{d}, \ldots, \frac{u_{\ell}}{d}$, respectively. Then the first $u_{1}$ partial computation paths generated for $a_{i}$ correspond to the first preference order, the next $u_{2}$ correspond to the second order, and so on. As a result, each total computation path corresponds to some preference profile. At the end of each computation path, the machine $\mathbb{M}$ checks (in deterministic polynomial time) whether the assignment is Pareto optimal for the corresponding preference profile, and accepts if and only if this is the case. It is straightforward to verify that the number of accepting computation paths of $\mathbb{M}$ is exactly the number $z$ such that the probability that the assignment is Pareto optimal is $\frac{z}{d^{n}}$. Therefore, we know that PO-PROBABILITY is in \#P.

We showed that when there are only a constant number of uncertain agents, we can compute the PO probability in polynomial time for the lottery model (Theorem 8). However, the order of the polynomial that upper bounds the running time of our proposed algorithm grows with the number of uncertain agents. In particular, when $k$ is the number of uncertain agents, and $\ell$ is the maximum number of possible preference orders for these uncertain agents, the running time of the algorithm outlined in the proof of Theorem 8 is $\Omega\left(\ell^{k}\right)$. We improve on this result by showing that there exists a fixed-parameter tractable algorithm that computes the PO probability for the lottery model - that is, an algorithm running in time $f(k)|I|^{c}$ for some computable function $f$ and some fixed constant $c$ independent of $k$, where $|I|$ denotes the input size. In other words, we show that the parameterized problem $k$-PO-PrOBABILITY, where the parameter is the number of uncertain agents, is fixed-parameter tractable for the lottery model. The proof involves an interesting reduction to counting homomorphisms.

Theorem 10. For the lottery model, $k$-PO-Probability can be solved in fixedparameter tractable time.

Proof. Take an arbitrary instance of the problem $k$-PO-ProbabiLity, consisting of agents $1, \ldots, n$, objects $o_{1}, \ldots, o_{n}$, and an assignment $\sigma$. Without 
loss of generality, assume that the assignment gives each agent $i$ the object $o_{i}$, and that the uncertain agents are agents $1, \ldots, k$. For each uncertain agent $i$, let $\succ_{i, 1}, \ldots, \succ_{i, u_{i}}$ denote the different possible preferences for agent $i$.

Additionally, assume without loss of generality that for each of the uncertain agents $1, \ldots, k$, each of the possible preferences for these agents occurs with probability $\frac{\ell}{d}$, where the numerator $\ell$ can vary between different agents and different possible preferences, but where the denominator $d$ is common among all agents and all possible preferences. In other words, all probabilities mentioned in the instance are rational numbers that share a common denominator $d$. If this were not the case, we could straightforwardly transform the instance in polynomial time to an equivalent instance that does satisfy this property.

Also, assume without loss of generality that there exists no trading cycle that involves only the agents $o_{k+1}, \ldots, o_{n}$. If this were the case, the assignment is Pareto optimal with probability zero, and we can filter out such trivial instances using a polynomial-time preprocessing procedure.

We now show how to compute the probability that the given assignment is Pareto optimal in fixed-parameter tractable time. Our computation will proceed in three stages:

(1) We construct a directed graph $G$ with $O\left(k u 2^{k^{2}}\right)$ vertices, where the edges are weighted. Here $u$ denotes the maximum number of possible preferences for any uncertain agent.

(2) We count the number of homomorphisms $f$ of a directed path $P_{2 k+2}$ of length $2 k+2$ to this graph $G$, where each homomorphism is counted multiple times according to (the product of) the weights on the edges in $f\left(P_{2 k+2}\right)$. This counting can be done in polynomial time using an extension of a known algorithm $[17,18]$.

(3) We divide the weighted total number of homomorphisms of $P_{2 k+2}$ to $G$ by the number $d^{k}$ to obtain the probability that the given assignment is Pareto optimal.

We begin with phase (1), and we construct the weighted, directed graph $G$. Let $\Pi=\left\{o_{1}, \ldots, o_{k}\right\}^{2}$ be the set of all possible pairs $\left(o_{i}, o_{j}\right)$ of objects among $o_{1}, \ldots, o_{k}$. We define the set $V$ of vertices of $G$ as follows. First, we define an auxiliary set $V^{\prime}$ :

$$
V^{\prime}=\{1, \ldots, k+1\} \cup\left\{\left(i, \succ_{i, j}\right) \mid i \in[k+1], j \in\left[u_{i}\right]\right\} .
$$

Then, we define the set $V$ of vertices as follows:

$$
V=\{s, t\} \cup\left\{\left(v^{\prime}, \Pi^{\prime}\right) \mid v^{\prime} \in V^{\prime}, \Pi^{\prime} \subseteq \Pi\right\} .
$$

That is, the graph $G$ has vertices $s$ and $t$, and $2^{k^{2}}$ copies of each element in $V^{\prime}$ (one for each $\Pi^{\prime} \subseteq \Pi$ ). Intuitively, the vertices $s$ and $t$ will act as source and target for each homomorphism of $P_{2 k+2}$ to $G$.

The sets $\Pi^{\prime} \subseteq \Pi$ will intuitively be used to memorize the 'trading paths' (i.e., paths in the trading cycle graph) that result from particular choices of the 
preference orders $\succ_{i, j}$ chosen for the agents $1, \ldots, k$. That is, each $\left(o_{i}, o_{j}\right) \in \Pi^{\prime}$ corresponds to a path from $o_{i}$ to $o_{j}$ in the directed graph with vertices $o_{1}, \ldots, o_{n}$ where there is an edge from $o_{i^{\prime}}$ to $o_{i^{\prime \prime}}$ if and only if agent $i^{\prime}$ prefers object $o_{i^{\prime \prime}}$ to object $o_{i^{\prime}}$.

We construct the set $E$ of (weighted and directed) edges as follows. This is where the agents' preference orders $\succ_{i, j}$ are encoded into the graph $G$-in particular, under (ii) and (iii).

(i) We add an edge with weight 1 from $s$ to $(1, \emptyset)$.

(ii) For each $i \in[k]$, each $j \in\left[u_{i}\right]$, and each $\Pi^{\prime} \subseteq \Pi$, we add an edge from $\left(i, \Pi^{\prime}\right)$ to $\left(i, \succ_{i, j}, \Pi^{\prime}\right)$. This edge has weight $\ell$, where the possible preference order $\succ_{i, j}$ for agent $i$ occurs with probability $\frac{\ell}{d}$.

(iii) For each $i \in[k]$, each $j \in\left[u_{i}\right]$, and each $\Pi^{\prime} \subseteq \Pi$, we add an edge with weight 1 from $\left(i, \succ_{i, j}, \Pi^{\prime}\right)$ to the vertex $\left(i+1, \Pi^{\prime \prime}\right)$, for some $\Pi^{\prime} \subseteq \Pi^{\prime \prime} \subseteq$ $\Pi$. The choice of $\Pi^{\prime \prime}$ is determined as follows. Consider the following graph $G_{\Pi^{\prime}, \succ i, j}$. The vertices of this graph are $o_{1}, \ldots, o_{n}$. For each pair $\left(o_{i^{\prime}}, o_{i^{\prime \prime}}\right)$ of vertices among $o_{k+1}, \ldots, o_{n}$, there is an edge from $o_{i^{\prime}}$ to $o_{i^{\prime \prime}}$ if and only if agent $j$ prefers object $o_{i^{\prime \prime}}$ to object $o_{i^{\prime}}$. Moreover, for each $\left(o_{i^{\prime}}, o_{i^{\prime \prime}}\right) \in \Pi^{\prime}$, we add an edge from $o_{i^{\prime}}$ to $o_{i^{\prime \prime}}$. Finally, for each agent $o_{i^{\prime}}$ among $o_{k+1}, \ldots, o_{n}$, we add an edge from $o_{i}$ to $o_{i^{\prime}}$ if and only if $o_{i^{\prime}} \succ_{i, j} o_{i}$. We then let $\Pi^{\prime \prime} \subseteq \Pi$ be the set of all pairs $\left(o_{i^{\prime}}, o_{i^{\prime \prime}}\right)$ such that there is a path from $o_{i^{\prime}}$ to $o_{i^{\prime \prime}}$ in $G_{\Pi^{\prime}, \succ i, j}$. Clearly, $\Pi^{\prime} \subseteq \Pi^{\prime \prime}$.

(iv) For each $\Pi^{\prime} \subseteq \Pi$ such that $\left(o_{i}, o_{i}\right) \notin \Pi^{\prime}$ for all $i$ among $1, \ldots, k$, we add an edge with weight 1 from $\left(k+1, \Pi^{\prime}\right)$ to $t$.

Clearly, any homomorphism $f$ from the directed path $P_{2 k+2}$ of length $2 k+2$ to $G$ must map the first vertex of the path to $s$ and the last vertex of the path to $t$. Each such homomorphism must map the $(2 i)$-th vertex of the path to some vertex $\left(i, \Pi^{\prime}\right)$ and the $(2 i+1)$-th vertex of the path to some vertex $\left(i, \succ_{i, j}, \Pi^{\prime}\right)$. Also, the $(2 k+2)$-th vertex of the path must be mapped to some vertex $\left(k+1, \Pi^{\prime}\right)$ where $\Pi^{\prime}$ contains no pair $\left(o_{i}, o_{i}\right)$. These observations follows directly from the construction of $G$.

Moreover, each homomorphism $f^{\prime}$ from the directed path $P_{2 k+1}$ of length $2 k+1$ to $G$ that maps the first vertex of the path to $s$ is uniquely determined by some series of choices $\succ_{1, j_{1}}, \ldots, \succ_{k, j_{k}}$ for the possible preferences of the uncertain agents $1, \ldots, k$. We argue that such a homomorphism $f^{\prime}$ can be extended to a homomorphism $f$ from $P_{2 k+2}$ to $G$ if and only if the corresponding preferences $\succ_{1, j_{1}}, \ldots, \succ_{k, j_{k}}$ do not lead to a trading cycle. The homomorphism $f^{\prime}$ maps the $(2 k+2)$-th vertex of the path to some pair $\left(k+1, \Pi^{\prime}\right)$. Here $\Pi^{\prime}$ is the set of pairs $\left(o_{i}, o_{j}\right) \in\left\{o_{1}, \ldots, o_{k}\right\}^{2}$ such that the preferences $\succ_{1, j_{1}}, \ldots, \succ_{k, j_{k}}$ lead to a trading path from $o_{i}$ to $o_{j}$. By our assumption that there exists no trading cycle that involves only the agents $o_{k+1}, \ldots, o_{n}$, we know that the set $\Pi^{\prime}$ contains some pair $\left(o_{i}, o_{i}\right)$ if and only if there exists a trading cycle. Therefore, by construction of the edges between $\left(k+1, \Pi^{\prime}\right)$ and $t$, we know that the choices $\succ_{1, j_{1}}, \ldots, \succ_{k, j_{k}}$ of preferences for the agents $1, \ldots, k$ 
that make the assignment Pareto optimal are in one-to-one correspondence with the homomorphisms $f$ from $P_{2 k+2}$ to $G$.

We count each such homomorphism $f$ in a weighted fashion as followsthis is phase (2). Take a homomorphism $f$ from $P_{2 k+2}$ to $G$. Its weight in the grand total is the product of the weights for each edge in $f\left(P_{2 k+2}\right)$. The only edges in $f\left(P_{2 k+2}\right)$ that have weigth $>1$ are edges from $\left(i, \Pi^{\prime}\right)$ to $\left(i, \succ_{i, j}\right.$ ,$\left.\Pi^{\prime}\right)$. Such an edge has weight $\ell$, where the probability that $\succ_{i, j}$ occurs is $\frac{\ell}{d}$. From this, it is straightforward to verify that the total weighted sum of all homomorphisms is equal to $p \cdot d^{k}$, where $p$ is the probability that the given assignment is Pareto optimal. Therefore, in order to compute $p$, we only need to take the weighted sum of all homomorphisms, and divide it by $d^{k}$ - this is phase (3) of the algorithm.

All that remains is to show how we can compute the weighted sum of all homomorphisms $f$ from $P_{2 k+2}$ to $G$ in polynomial time. We can do this by extending a known polynomial-time algorithm to count the number of homomorphisms of a graph whose treewidth is bounded by a fixed constant into another graph [18, Theorem 14.7]. Since paths have treewidth 1, counting the number of homomorphisms from a path to another graph can be done in polynomial time using this algorithm. This algorithm uses a dynamic programming approach to count the number of homomorphisms. This dynamic programming technique can straightforwardly be extended to take into account the weights of the homomorphisms. (We omit a detailed description of the extended algorithm.)

This concludes our proof that $k$-PO-ProBABILITY can be solved in fixedparameter tractable time for the lottery model.

\section{Compact Indifference Model}

In this section, we turn to the compact indifference model. Any compact indifference preference profile admits a possibly PO assignment that can be computed as follows: break the ties arbitrarily and then run serial dictatorship. What about the existence of certainly PO assignments? Although the compact indifference model is one of the most restricted and structured uncertainty models we consider, ExistCERTAINLYPO-AssignMENT is NP-complete for this model.

Theorem 11. For the compact indifference uncertainty model, ExISTCertainlyPO-Assignment is NP-complete.

Proof. ExistsCertainlyPO-Assignment is in NP because it can be checked in polynomial time whether a given assignment is certainly PO or not (Corollary 1).

We show hardness by reducing from the SerialDictatorshipFeasibilITY problem. Let $\left(N, O, \succ^{\prime}, i, o\right)$ be an instance of this problem, where $N=$ $\{1, \ldots, n\}$ and $O=\left\{o_{1}, \ldots, o_{n}\right\}$. W.l.o.g., suppose that $i=1$. We construct 
an instance $\left(N^{\prime}, O^{\prime}, \succ\right)$ of the compact indifference uncertainty model as follows. Let $o^{\prime}, o^{\prime \prime}$ be fresh items not appearing in $O$, let $O^{\prime}=O \cup\left\{o^{\prime}, o^{\prime \prime}\right\}$, and let $N^{\prime}=N \cup\left\{1^{\prime}, n+1\right\}$.

Let $O_{1, \succ^{\prime} o}$ be the items that agent 1 prefers over item $o$ with respect to $\succ_{1}^{\prime}-$ i.e., those items $o_{\ell}$ such that $o_{\ell} \succ_{1}^{\prime} o$. Moreover, let $O_{1, \prec^{\prime} o}$ be the items that agent 1 likes less than item $o$-i.e., $O_{1, \prec^{\prime} o}=O \backslash\left(\{o\} \cup O_{1, \succ^{\prime} o}\right)$. Also, for each $1<j \leq n$, let $j_{1}, \ldots, j_{n}$ be the indices of items such that $o_{j_{1}} \succ_{j}^{\prime} \cdots \succ_{j}^{\prime} o_{j_{n}}$.

We define the preferences of the agents in $N^{\prime}$ over the items in $O^{\prime}$ as follows:

- Agent 1: $\left(O_{1, \succ^{\prime} o} \cup\left\{o^{\prime}\right\}\right) \succ_{1} o \succ_{1}\left(O_{1, \prec^{\prime} o} \cup\left\{o^{\prime \prime}\right\}\right)$.

- Agent $1^{\prime}:\left(O_{1, \succ^{\prime} o} \cup\left\{o^{\prime}\right\}\right) \succ_{1^{\prime}} o \succ_{1^{\prime}}\left(O_{1, \prec^{\prime} o} \cup\left\{o^{\prime \prime}\right\}\right)$.

- Agents $1<j \leq n: o_{j_{1}} \succ_{j} \cdots \succ_{j} o_{j_{n}} \succ_{j}\left\{o^{\prime}, o^{\prime \prime}\right\}$.

- Agent $n+1: O^{\prime}$ (one big tie).

We show that there is a permutation $\pi$ of the agents in $N$ under which agent $i=1$ gets item $o$ when serial dictatorship is run on $\left(N, O, \succ^{\prime}\right)$ if and only if there is an assignment that is certainly $\mathrm{PO}$ for $\left(N^{\prime}, O^{\prime}, \succ\right)$.

$(\Rightarrow)$ Suppose that there is a permutation $\pi$ of the agents in $N$ under which agent $i=1$ gets item $o$ when serial dictatorship is run on $\left(N, O, \succ^{\prime}\right)$. Let $M$ denote this PO assignment. Let $M^{\prime}=M+\left\{\left(1^{\prime}, o^{\prime}\right),\left(n+1, o^{\prime \prime}\right)\right\}$, that is, $M^{\prime}$ extends $M$ by awarding the new items $o^{\prime}$ and $o^{\prime \prime}$ to new agents $1^{\prime}$ and $n+1$ respectively. It is easy to see that $M^{\prime}$ is certainly $\mathrm{PO}$ for $\left(N^{\prime}, O^{\prime}, \succ\right)$.

$(\Leftarrow)$ Conversely, suppose that there is an assignment $M^{\prime}$ that is certainly PO for $\left(N^{\prime}, O^{\prime}, \succ\right)$. Then it can be proved that following statements are true w.r.t. $M^{\prime}$.

(i) Claim: Item $o^{\prime \prime}$ must go to $n+1$.

Proof: Otherwise, under a complete linear order extension of $\succ$ in which $n+1$ ranks $o^{\prime \prime}$ at the top, and the agent who has got $o^{\prime \prime}$ ranks it at the bottom, both agent benefit by swapping their assigned items.

(ii) Claim: Agents 1 and $1^{\prime}$ cannot get an item from their last ties.

Proof: Assume otherwise, then 1 and $1^{\prime}$ get items say $o_{1}$ and $o_{2}$ from their last tie. Recall that we already argued that $n+1$ gets $o^{\prime \prime}$. Under a complete linear order extension in which 1 prefers $o_{2}$ to $o_{1}, 2$ prefers $o^{\prime \prime}$ to $o_{2}$, and $n+1$ prefers $o_{1}$ to $o^{\prime \prime}$, agents $1,1^{\prime}$ and $n+1$ all benefit by trading their assigned items $\left(\left\langle o_{1}, 1, o_{2}, 1^{\prime}, o^{\prime \prime}, n+1, o_{1}\right\rangle\right.$ is a trading cycle).

(iii) Claim: Agents 1 and $1^{\prime}$ cannot both get an item from their first ties. Therefore one of them, say 1 gets $o$, and the other one, say $1^{\prime}$, gets an item from her first tie. In fact, since 1 and $1^{\prime}$ have exactly the same preferences under $\succ$, we can assume w.l.o.g. that 1 gets $o$ in $M^{\prime}$.

Proof: Assume otherwise, then 1 and $1^{\prime}$ get items say $o_{1}$ and $o_{2}$ from their first tie. Under a complete linear order extension in which 1 prefers $O_{2}$ to $o_{1}$ and 2 prefers $o_{1}$ to $o_{2}$ both agents benefit from swapping their assigned items. 
(iv) Claim: Agent $1^{\prime}$ must get item $o^{\prime}$.

Proof: We already argued that $1^{\prime}$ gets an item from her first tie. Assume that $1^{\prime}$ does not get $o^{\prime}$. Thus $1^{\prime}$ gets another item $o_{1}$ from her first tie, and another agent $j$ gets $o^{\prime}$. Agent $j$ cannot be 1 or $n+1$, therefore by the construction of $\succ$ she ranks $o^{\prime}$ in a tie with $o^{\prime \prime}$ at the bottom of her preferences. Under any complete linear order extension in which $1^{\prime}$ prefers $o^{\prime}$ to $o_{1}$, both $1^{\prime}$ and $j$ benefit by swapping their assigned items.

We have established so far that in $M^{\prime}$ items $o$ and $o^{\prime}$ go to agents 1 and $1^{\prime}$ respectively, and item $o^{\prime \prime}$ goes to agent $n+1$. Let $M$ be the assignment $M^{\prime}$ restricted to the original agents. It is clear that since $M^{\prime}$ is certainly PO for $\succ$, hence $M$ is PO for $\succ^{\prime}$. Furthermore, agent $i=1$ is awarded item $o$ in $M$. Therefore, by Fact 1, there is a permutation of agents in $N$ under which $i=1$ gets $O$ when serial dictatorship is run on $\left(N, O, \succ^{\prime}\right)$.

Theorem 11 gives us the following corollary.

Corollary 6. For the compact indifference uncertainty model, AssignMENTWithHighestPO-ProB is NP-hard.

Finally, we show that PO-ProBABILITY is \#P-complete for the compact indifference model.

Theorem 12. For the compact indifference uncertainty model, POProbability is \#P-complete.

Proof. It is straightforward to show that PO-Probability is in \#P. We show \#P-hardness by reducing from the \#P-complete problem Monotone-\#2SAT that is defined as follows: count the number of satisfying assignments for a 2CNF formula that contains no negation [31].

Let $\varphi$ be a monotone $2 \mathrm{CNF}$ formula with clauses $c_{1}, \ldots, c_{m}$ and variables $x_{1}, \ldots, x_{n}$. We construct an instance of PO-ProbABILITY as follows. Consider agents $1, \ldots, 2 n$, and items $o_{1}, \ldots, o_{2 n}$ and take the assignment $\omega$ where $\omega(i)=o_{i}$ for all $1 \leq i \leq 2 n$. Agents' preferences are constructed as follows. Take an arbitrary $1 \leq i \leq n$. Consider the set $\left\{j_{1}, \ldots, j_{u}\right\}$ of indices $j$ such that the clause $\left(x_{i} \vee x_{j}\right)$ occurs in $\varphi$. Suppose that $j_{1}<j_{2}<\cdots<j_{u}$, in order to fix an (arbitrary) order over these indices. Then agent $i$ has the following preference: $\left(o_{n+i}, o_{i}\right) \succ_{i} \cdots$, where the remaining items appear in arbitrary order. Moreover, agent $n+i$ has the following preference: $o_{j_{1}} \succ_{n+i} \cdots \succ_{n+i} o_{j_{u}} \succ_{n+i} o_{n+i} \succ_{n+i} \cdots$, where the remaining items appear in arbitrary order. Note that agents $n+1, \ldots, 2 n$ have certain preferences.

The instantiations of linear preferences to uncertain agents $1, \ldots, n$ correspond one-to-one to truth assignments to variables $x_{1}, \ldots, x_{n}$ : for each $1 \leq i \leq$ $n$, setting the preferences of agent $i$ to $o_{i} \succ_{i} o_{n+i} \succ_{i} \cdots$ corresponds to setting $x_{i}$ to true, and setting agent $i$ 's preferences to $o_{n+i} \succ_{i} o_{i} \succ_{i} \cdots$ corresponds to setting $x_{i}$ to false.

We show that a truth assignment $\alpha$ to the variables $x_{1}, \ldots, x_{n}$ satisfies $\varphi$ if and only if the corresponding profile of linear preferences for the agents $1, \ldots, 2 n$ leads the assignment $\omega$ to be PO. 
$(\Rightarrow)$ Suppose that $\alpha$ satisfies $\varphi$. We show that the assignment $\omega$ admits no trading cycles under the profile corresponding to $\alpha$. Suppose, to derive a contradiction, that $\omega$ admits a trading cycle. By construction of the preferences of the agents, any trading cycle must involve the sequence $\left\langle o_{i}, i, o_{n+i}, n+\right.$ $\left.i, o_{j}, j, o_{n+j}, n+j, o_{i}\right\rangle$. That is, agent $i$ must prefer $o_{n+i}$ over $o_{i}$, agent $j$ must prefer $o_{n+j}$ over $o_{j}$, and agent $n+i$ must prefer $o_{j}$ over $o_{n+i}$. The former two statements are the case if and only if $\alpha$ sets both $x_{i}$ and $x_{j}$ to false. The latter statement is the case if and only if $\left(x_{i} \vee x_{j}\right)$ is a clause of $\varphi$. This is a contradiction to our assumption that $\alpha$ satisfies $\varphi$. Thus, we can conclude that $\omega$ is PO under the profile corresponding to $\alpha$.

$(\Leftarrow)$ Conversely, suppose that $\alpha$ does not satisfy $\varphi$. That is, there is a clause $\left(x_{i} \vee x_{j}\right)$ of $\varphi$ such that $\alpha$ sets both $x_{i}$ and $x_{j}$ to false. We show that the assignment $\omega$ admits a trading cycle under the profile corresponding to $\alpha$. Since $\alpha$ sets $x_{i}$ and $x_{j}$ to false, we know that $o_{n+i} \succ_{i} o_{i}$ and $o_{n+j} \succ_{j} o_{j}$. Moreover, since $\left(x_{i} \vee x_{j}\right)$ is a clause of $\varphi$, we know that $o_{j} \succ_{n+i} o_{n+i}$ and $o_{i} \succ_{n+j}$ $o_{n+j}$. Thus, $\left\langle o_{i}, i, o_{n+i}, n+i, o_{j}, j, o_{n+j}, n+j, o_{i}\right\rangle$ is a trading cycle under this preference profile, and hence $\omega$ is not PO.

Each possible preference profile occurs with probability $2^{-n}$. The number of satisfying truth assignments $\varphi$ is then exactly equal to $2^{n}$ times the probability that assignment $\omega$ is PO. Thus, PO-ProBABILITy is \#P-hard.

\section{Pairwise Model}

In this section, we present results for the pairwise uncertainty model. A possibly PO assignment exists for the pairwise model if the 'certainly preferred' relation of each agent is acyclic: one can derive a possible linear order consistent with the certainly preferred relation and then run serial dictatorship. In the presence of cycles, however, there is no guarantee that an instance admits a possibly PO assignment. The next example shows that under the pairwise model, if the certainly preferred relation is not acyclic, then there may not exist a possibly PO assignment.

Example 4. The pairwise preferences for the agents 1, 2 and 3 over the items $a, b$ and $c$ are as depicted. All the three agents have the same certainly preferred relations.

$$
\begin{array}{ll}
1: & a \succ_{1}^{\text {cert }} c, c \succ_{1}^{\text {cert }} b, b \succ_{1}^{\text {cert }} a \\
2: & a \succ_{2}^{\text {cert }} c, c \succ_{2}^{\text {cert }} b, b \succ_{2}^{\text {cert }} a \\
3: & a \succ_{3}^{\text {cert }} c, c \succ_{3}^{\text {cert }} b, b \succ_{3}^{\text {cert }} a
\end{array}
$$

Take assignment abc. It is not PO because it is Pareto dominated by bca. By symmetry no other assignment is PO either.

We show, with an argument similar to that of the proof of Theorem 6 or Theorem 11 that the problem of checking whether a PO assignment exists is NP-complete under pairwise preferences even in the absence of uncertainty. 
Theorem 13. Given an instance of the assignment problem with deterministic pairwise preferences, the problem of deciding if a PO assignment exists is NPcomplete.

Proof. The problem is in NP because it can be checked in polynomial time whether a given assignment is certainly PO (Corollary 1 ) which is equivalent to $\mathrm{PO}$ under deterministic pairwise preferences.

To prove NP-hardness, we reduce from the NP-complete problem SERIALDictatorshipFEasibiLity. Let $\left(N, O, \succ^{\prime}, i, o\right)$ be an instance of this problem, where $N=\{1, \ldots, n\}$ and $O=\left\{o_{1}, \ldots, o_{n}\right\}$. We construct an instance $\left(N^{\prime}, O^{\prime}, \succ\right)$ of the pairwise uncertainty model as follows. Let $o^{\prime}, o^{\prime \prime}$ be fresh items not appearing in $O$, let $O^{\prime}=O \cup\left\{o^{\prime}, o^{\prime \prime}\right\}$, and let $N^{\prime}=$ $N \cup\{n+1, n+2\}$.

- For agent $i$ :

- For each pair of items $o_{1}, o_{2}$ such that $o \notin\left\{o_{1}, o_{2}\right\}$, let $o_{1} \succ_{i}^{\text {cert }} o_{2}$ if and only if $o_{1} \succ_{i}^{\prime} o_{2}$.

- Let $o \succ_{i}^{\text {cert }} o^{\prime}, o \succ_{i}^{\text {cert }} o^{\prime \prime}$ and $o^{\prime} \succ_{i}^{\text {cert }} o^{\prime \prime}$.

- For each item $o_{1} \in O \backslash\{o\}$, let $o_{1} \succ_{i}^{\text {cert }} o, o_{1} \succ_{i}^{\text {cert }} o^{\prime}$, and $o_{1} \succ_{i}^{\text {cert }} o^{\prime \prime}$ if and only if $o_{1} \succ_{i}^{\prime} o$.

- For each item $o_{1} \in O \backslash\{o\}$, let $o \succ_{i}^{\text {cert }} o_{1}, o^{\prime} \succ_{i}^{\text {cert }} o_{1}$, and $o^{\prime \prime} \succ_{i}^{\text {cert }} o_{1}$ if and only if $o \succ_{i}^{\prime} o_{1}$.

- For each agent $j \in N \backslash\{i\}$ :

- For each pair of items $o_{1}, o_{2}$ such that $o \notin\left\{o_{1}, o_{2}\right\}$, let $o_{1} \succ_{j}^{\text {cert }} o_{2}$ if and only if $o_{1} \succ_{j}^{\prime} o_{2}$.

- Let $o \succ_{j}^{\text {cert }} o^{\prime}, o^{\prime} \succ_{j}^{\text {cert }} o^{\prime \prime}$ and $o^{\prime \prime} \succ_{j}^{\text {cert }} o$.

- For each item $o_{1} \in O \backslash\{o\}$, let $o_{1} \succ_{j}^{\text {cert }} o, o_{1} \succ_{j}^{\text {cert }} o^{\prime}$, and $o_{1} \succ_{j}^{\text {cert }} o^{\prime \prime}$ if and only if $o_{1} \succ_{j}^{\prime} o$.

- For each item $o_{1} \in O \backslash\{o\}$, let $o \succ_{j}^{\text {cert }} o_{1}, o^{\prime} \succ_{j}^{\text {cert }} o_{1}$, and $o^{\prime \prime} \succ_{j}^{\text {cert }} o_{1}$ if and only if $o \succ_{j}^{\prime} o_{1}$.

- For both agents $j \in\{n+1, n+2\}$ :

- For each pair of items $o_{\ell}, o_{\ell^{\prime}} \in O \backslash\{o\}$, let $o_{\ell} \succ_{j}^{\text {cert }} o_{\ell^{\prime}}$ if and only if $\ell>\ell^{\prime}$.

- For each item $o_{\ell} \in O \backslash\{o\}$, let $o \succ_{j}^{\text {cert }} o_{\ell}, o^{\prime} \succ_{j}^{\text {cert }} o_{\ell}$, and $o^{\prime \prime} \succ_{j}^{\text {cert }} o_{\ell}$.

- Let $o \succ_{j}^{\text {cert }} o^{\prime}, o^{\prime} \succ_{j}^{\text {cert }} o^{\prime \prime}$ and $o^{\prime \prime} \succ_{j}^{\text {cert }} o$.

Intuitively, the (linear) preference $\succ_{i}^{\text {cert }}$ is obtained from $\succ_{i}^{\prime}$ by replacing $o$ with $o \succ o^{\prime} \succ o^{\prime \prime}$. The preference $\succ_{j}^{\text {cert }}$ for each $j \in N \backslash\{i\}$ is obtained from $\succ_{j}^{\prime}$ by replacing $o$ with the cycle $o \succ o^{\prime} \succ o^{\prime \prime} \succ o$. Finally, the preferences $\succ_{n+1}^{\text {cert }}$ and $\succ_{n+2}^{\text {cert }}$ are constructed by having the cycle $o \succ o^{\prime} \succ o^{\prime \prime} \succ o$ on the top, 
followed by the items in $O \backslash\{o\}$ (in arbitrary order). Note that all these pairwise preferences are deterministic.

We show that there is a permutation of agents $\pi$ under which agent $i$ gets item $o$ when serial dictatorship is run on $\left(N, O, \succ^{\prime}\right)$ if and only if there is an assignment that is $\mathrm{PO}$ for $\left(N^{\prime}, O^{\prime}, \succ\right)$.

$(\Rightarrow)$ Suppose that there is a permutation of agents $\pi$ under which agent $i$ gets item $o$ when serial dictatorship is run on $\left(N, O, \succ^{\prime}\right)$ (resulting in assignment $\omega$ ). Construct the permutation $\pi^{\prime}$ for agents $N^{\prime}$ obtained from $\pi$ by placing the agents $n+1, n+2$ directly after agent $i$. Then $\operatorname{SD}\left(N^{\prime}, O^{\prime}, \succ, \pi^{\prime}\right)$ returns the assignment $\omega^{\prime}=\omega \cup\left\{n+1 \mapsto o^{\prime}, n+2 \mapsto o^{\prime \prime}\right\}$. This assignment $\omega^{\prime}$ is PO.

$(\Leftarrow)$ Suppose that there is an assignment $\omega^{\prime}$ for $\left(N^{\prime}, O^{\prime}, \succ\right)$ that is PO. By Theorem 3, we know that there exists a permutation $\pi^{\prime}$ of the agents in $N^{\prime}$ such that executing SD with this permutation on $\left(N^{\prime}, O^{\prime}, \succ\right)$ yields $\omega^{\prime}$. Let $\pi^{\prime}=$ $\left(\pi_{0}, i, \pi_{1}\right)$, i.e., $\pi_{0}$ is the sequence of agents appearing in $\pi$ before agent $i$, and $\pi_{1}$ is the sequence of agents appearing in $\pi$ after agent $i$. By construction of $\succ$, we know that $o$ is picked before $o^{\prime}$ and $o^{\prime \prime}$, because $o^{\prime}$ and $o^{\prime \prime}$ are dominated in every preference order whenever $o$ has not yet been picked. Moreover, we know that agent $i$ must pick item $o$, because whenever $o, o^{\prime}, o^{\prime \prime}$ have not yet been picked, item $o$ is dominated in every preference order except agent $i$ 's. Thus, after the agents in $\pi_{0}$ have picked the unique most preferred remaining item in their preference order, all items that agent $i$ prefers to item $o$ (in $\succ_{i}^{\prime}$ ) have been picked. Note that agents $n+1$ and $n+2$ are not in $\pi_{0}$, since as long as item $o$ is not picked both of these agents are confused (i.e. they don't have a most preferred item in their preference order). Thus, $\left(\pi_{0}, i\right)$ can be extended (by appending the rest of agents in $N$ in arbitrary order) to a permutation $\pi$ under which agent $i$ gets item $o$ when serial dictatorship is run on $\left(N, O, \succ^{\prime}\right)$ with permutation of agents $\pi$.

Since possibly PO and certainly PO assignments coincide with PO assignments for deterministic pairwise preferences, we get the following corollary.

Corollary 7. For the pairwise uncertainty model, ExistsPossiblyPOAssignment and ExistsCertainlyPO-Assignment are NP-complete even if pairwise preferences are all deterministic.

The proof of Theorem 13 exploits the fact that certainly preferred relations can be cyclic under the pairwise uncertainty model. What if we disallow cycles in certainly preferred relations? Our next result shows that checking whether a certainly PO assignment exists is NP-complete for instances of pairwise uncertainty model, even if the certainly preferred part of the preferences is acyclic.

Theorem 14. For the pairwise uncertainty model, ExistsCertainlyPOAssignment is NP-complete even if the certainly preferred part of the preferences is acyclic.

Proof. ExistsCertainlyPO-Assignment is in NP because it can be checked in polynomial time whether a given assignment is certainly PO or not (Corollary 1). 
To prove NP-hardness, we reduce from SERIalDictatorshipFeasibility problem. Let $\left(N, O, \succ^{\prime}, i, o\right)$ be an instance of this problem. We construct an instance of the pairwise uncertainty model $(N, O, \succ)$ as follows.

- For agent $i$ :

- For all pair of items $o_{t}$ and $o_{k}, o_{t} \neq o$ and $o_{k} \neq o$, let $o_{t} \succ_{i}^{\text {cert }} o_{k}$ if and only if $o_{t} \succ_{i}^{\prime} o_{k}$.

- For all items $o_{k}$ such that $o \succ_{i}^{\prime} o_{k}$, let $o \succ_{i}^{\text {cert }} o_{k}$

- For all items $o_{k}$ such that $o_{k} \succ_{i}^{\prime} o$, let $p\left(o \succ_{i} o_{k}\right)=0.5$. That is, $i$ prefers $O$ over $o_{k}$ with probability half, and prefers $o_{k}$ to $o$ with probability half as well. In fact we can replace 0.5 with any value in $(0,1)$.

- For each agent $j \in N \backslash\{i\}$ :

- For all pair of items $o_{t}$ and $o_{k}, o_{t} \neq o$ and $o_{k} \neq o$, let $o_{t} \succ_{j}^{\text {cert }} o_{k}$ if and only if $o_{t} \succ_{j}^{\prime} o_{k}$.

- For all items $o_{k}$ such that $o_{k} \succ_{j}^{\prime} o$, let $o_{k} \succ_{j}^{\text {cert } o}$

- For all items $o_{k}$ such that $o \succ_{j}^{\prime} o_{k}$, let $p\left(o_{k} \succ_{j} o\right)=0.5$. That is, $j$ prefers $o_{k}$ over $o$ with probability half, and prefers $o$ to $o_{k}$ with probability half as well. In fact we can replace 0.5 with any value in $(0,1)$.

Intuitively speaking, in the uncertain pairwise instance constructed above, each agent preserves the same certainly preferred relation as in $\succ^{\prime}$ for all pairs of items that do not include $o$. In addition, agent $i$ preservers the certainly preferred relation between $o$ and every item she likes less than $o$ under $\succ^{\prime}$, and every other agent preserves the certainly preferred relation between $o$ and every item she liked better than $o$ under $\succ^{\prime}$. Every other possible pairwise comparisons are assigned probability half. This way, we can ensure that there exists a linear realization of the preference profiles such that $i$ ranks $o$ at the top of her preference list, and every other agent ranks $o$ at the bottom of their preference lists. We denote this linear preference profile by $\succ^{*}$. Also, $\succ^{\prime}$ is one of the realizable preference profiles.

Our first observation is that an assignment is PO under profile $\succ^{*}$ only if $i$ receives $o$ in it (Observation 1).

We now prove that we have a yes instance of SERIALDictatorshipFEaSIBILITY if and only if our constructed uncertain pairwise instance admits a certainly PO assignment. Since SerialDictatorshipFeasibility is NPcomplete, this will entail that ExistsCertainlyPO-Assignment is NPcomplete.

Assume that there exists a certainly $\mathrm{PO}$ assignment $\omega$. Then, $\omega$ must be $\mathrm{PO}$ under $\succ^{*}$ implying that, by Observation 1, $i$ receives $o$ in $\omega$. Assignment $\omega$ must also be PO under profile $\succ^{\prime}$ which implies that there exists an assignment (i.e. 
$\omega)$ that is PO under profile $\succ^{\prime}$ and in which $i$ receives $o$. In light of Fact 1, this implies that there exists a serial dictatorship the outcome of which under profile $\succ^{\prime}$ is $\omega$. Hence, we have a yes instance of SerialDictatorshipFEasibility.

Now consider the case when we have a yes instance of SerialDictatorSHIPFEASIBILITY. This means that there is a permutation of agents $\pi$ under which $i$ gets $o$ when serial dictatorship is run. Let us call this assignment by $\omega$. Due to Fact 1, $\omega$ is PO under preference profile $\succ^{\prime}$. It remains to show that $\omega$ is PO under all preference profiles that are realizable from $\succ$. Due to Theorem 3 , it is sufficient to prove that for all realizable preference profiles, there exists a corresponding permutation of agents under which the outcome of serial dictatorship is $\omega$.

In fact, we show that $\operatorname{SD}\left(N, O, \succ^{r}, \pi\right)=\omega$ for all realizable preference profiles $\succ^{r}$. The proof is by induction on the number of rounds in SD, and by showing that at the end of any given round $k$, all agents $\pi(1) \ldots \pi(k)$ are assigned the same items as in $\omega$.

Round 1: Either the first agent in the permutation is $i$ or not.

- If $\pi(1)=i$ : It must be that $i$ ranks $o$ at the top of $\succ_{i}^{\prime}$. Therefore, $o \succ_{i}^{\text {cert }} o_{k}$ for all items $o_{k} \neq o$. Hence in all realizable preference profiles $\succ_{i}^{r}, i$ prefers $o$ to all other items and there $\operatorname{SD}\left(N, O, \succ^{r}, \pi\right)$ assigns $o=\omega(i)$ to $i$.

- If $\pi(1)=j, j \neq i$ : First note that $j$ cannot have $o$ at the top of $\succ_{j}^{\prime}$ or $\omega(j)=o$, a contradiction. Therefore, there is another item, say $o^{\prime}$, at the top of $\succ_{j}^{\prime}$ and $\omega(j)=o^{\prime}$. By the construction of $\succ_{j}, j$ prefers $o^{\prime}$ to all other items in every realizable preference profile $\succ_{j}^{r}$, and hence $\operatorname{SD}\left(N, O, \succ^{r}, \pi\right)$ assigns $o^{\prime}=\omega(j)$ to $j$.

Assume that the induction claim holds for $k \geq 1$. We show that it holds for $k+1$.

Round $k+1$ : either the $k+1$ 'th agent in the permutation is $i$ or not.

- If $\pi(k+1)=i$ : When serial dictatorship is executed given $\succ^{\prime}$ and the permutation of the agents $\pi, i$ is assigned $o$. This implies that $o$ is still available, and all items $i$ prefers to $o$ under $\succ^{\prime}$ are taken. As the assignment of all agents who appeared earlier than $\pi(k+1)=i$ are the same under both $\succ^{\prime}$ and $\succ^{r}$, therefore the set of remaining items $T$ is the same under both preference profiles and includes $o$. Therefore, by the construction of $\succ_{i}, o \succ_{i}^{\text {cert }} o_{k}$ for all $o_{k} \in T$, and hence $i$ prefers $o$ to all items in $T$ under all realized preference profiles $\succ_{i}^{r}$. Therefore $\operatorname{SD}\left(N, O, \succ^{r}, \pi\right)$ assigns $o=\omega(i)$ to $i$.

- If $\pi(k+1)=i, j \neq i$ : Agent $j$ is assigned an item that is not $o$, say $o^{\prime}$, in $\omega$. This means that when serial dictatorship is executed given $\succ^{\prime}$ and the permutation of the agents $\pi, o^{\prime}$ is available when it is $j$ 's turn, and all items $j$ prefers to $o^{\prime}$ are taken. This implies that if $o$ is not taken yet, then 
it must be the case that $o^{\prime} \succ_{j}^{\prime} o$ (Observation 2). As the assignment of all agents who appeared earlier than $\pi(k+1)=i$ are the same under both $\succ^{\prime}$ and $\succ^{r}$, therefore the set of remaining items $T$ is the same under both preference profiles and includes $o^{\prime}$. By the construction of $\succ_{j}$ and Observation 2, $o^{\prime} \succ_{j}^{\text {cert }} o_{k}$ for all $o_{k} \in T$, and hence $j$ prefers $o^{\prime}$ to all items in $T$ under all realized preference profiles $\succ_{j}^{r}$. Therefore $\operatorname{SD}\left(N, O, \succ^{r}, \pi\right)$ assigns $o^{\prime}=\omega(j)$ to $j$.

Thus $\omega$ is PO under all possibly realizable preference profiles, and this completes the proof.

Theorem 14 gives us the following corollary.

Corollary 8. For the pairwise uncertainty model, AssignmentWithHighestPO-ProB is NP-hard even if the certainly preferred part of the preferences is acyclic.

Theorem 15. For the pairwise model, PO-Probability is \#P-complete.

Proof. It is straightforward to show that PO-Probability is in \#P. Hardness for \#P can be shown by following the reduction used in the proof of Theorem 12 . In this reduction, each agents either has a certain linear preference, or a preference where they are indifferent between their two most preferred items (and have a certain linear preference over all other items). These preferences can straightforwardly be expressed in the pairwise model, hence PO-PROBABILITY is \#P-hard under this model as well.

\section{Ranking Model}

In this section, we consider the ranking uncertainty model. In the ranking model, each agent $i$ reports a bistochastic matrix $M_{i}$ of size $n \times n$. The rows of the matrix correspond to indices of items and the columns to the rank of the items. Note that if the matrix is a permutation matrix (with only 0-1 entries), then the ranking model degenerates to a linear preference. We say that a permutation matrix $P$ is consistent with a bistochastic matrix $Q$ if for each $P_{i j}=1$, we have that $Q_{i j}>0$. We say that a linear preference $\succ_{i}$ is consistent with a ranking preference if the permutation matrix representing $\succ_{i}$ is consistent with the ranking preference matrix. For any ranking preference matrix $M_{i}$ for agent $i$, we will also consider a corresponding 'consistency graph' which is a bipartite graph $(O \cup R, E)$ where $R=\{1, \ldots, n\}$ is the set of possible ranks and $(o, r) \in E$ if $i$ expresses non-zero probability for $o$ to be in rank $r$.

By Birkhoff's theorem [22], any bistochastic matrix can be represented as a convex combination of at most quadratic number of permutation matrices. ${ }^{4}$ Hence, a ranking preference profile can always be represented as a lottery preference profile. This representation is not necessarily unique; that is, a ranking

${ }^{4}$ We say that the bistochastic matrix is decomposed into permutation matrices. 
preference profile may have several or possibly even exponentially many lottery preference profile representation. Due to this reason, it is not clear whether the PO probability of a matching is well-defined under the ranking model. In view of this we clarify the definitions of possibly PO and certainly PO that we are using for the ranking model. We say that an assignment is certainly $\mathrm{PO}$ under the ranking model if it is certainly $\mathrm{PO}$ for all lottery preference profiles that represent the ranking preference profile. Likewise, we say that an assignment is possibly PO under the ranking model if it is possibly PO for some lottery preference profile that represents the ranking preference profile. Next, we argue that Corollaries 1 and 2 regarding IsPO-ProBABILITYONE and IsPO-ProbabilityNonZero hold under the ranking model. In order to do so, we will show that "possibly preferred" (and hence "certainly preferred") and "possibly most preferred item in a set" can be checked in polynomial time.

Given a ranking preference profile $\succ$, we say that $b \succ_{i}^{\text {cert }} c$ if $b \succ_{i}^{\prime} c$ in each linear preference profile $\succ^{\prime}$ that is in the support of some lottery preference profile that represents $\succ$. Note that a linear preference is in the support of some lottery preference that represents a ranking preference if and only if the linear preference is consistent with the ranking preference. The negation of $b \succ_{i}^{\text {cert }} c$ holds if there exist $j, k \in[n]$ such that $j<k$ and there is a linear preference consistent with the ranking preferences in which $b$ gets rank $k$ and $c$ gets rank $j$. The relation $b \nsucc_{i}^{\text {cert }} c$ can be tested by checking whether there exist $j, k \in[n]$ such that $j<k$ and the consistency graph corresponding to the ranking preferences admits a perfect matching in which $(b, k)$ and $(c, j)$ are part of the matching.

Given a ranking preference profile $\succ$, we say that $a$ is a possibly most preferred item of agent $i$ among a set of items $O^{\prime}$, if there is a linear preference consistent with $\succ$ in which $a$ is the most preferred item in set $O^{\prime}$. The latter can be checked in polynomial time as follows. For each possible rank $j$ for item $a$, we construct a corresponding graph $G_{j}=(O \cup R, E)$ which is derived from the consistency graph by removing all edges involving $a$ except $(a, j)$, as well as removing all edges $\left(o^{\prime}, k\right)$ where $o^{\prime} \in O^{\prime} \backslash\{a\}$ and $k \leq j$. Then $a$ is a possibly most preferred item of $i$ in $O^{\prime}$ if and only if there exists a perfect matching for some $G_{j}$.

For the ranking model, a possibly PO assignment always exists and can be computed by finding a linear preference profile consistent with the ranking model and then running serial dictatorship.

Theorem 16. For the ranking uncertainty model, a possibly PO assignment always exists and can be computed in polynomial time.

Proof. Note that in the ranking model, each agent $i$ reports a bistochastic matrix $M_{i}$ of size $n \times n$. Since the matrix is bistochastic, it admits at least one permutation matrix consistent with $M_{i}$. Such a permutation matrix is a valid possible deterministic preference of agent $i$ and it can be computed in polynomial time via reduction to finding a perfect matching. Thus we can find for each agent a possible linear order. Once we compute a linear order for each 
agent, we can run serial dictatorship on the resultant linear order profile to find a possibly $\mathrm{PO}$ assignment.

On the other hand, just like the other four models, ExistsCERTAINLYPOAssignment is NP-complete under the ranking model. The proof is an adaptation of the proof of Theorem 7 .

Theorem 17. For the ranking model, ExistsCertainlyPO-Assignment is NP-complete.

Proof. The problem ExistsCertainlyPO-Assignment is in NP because it can be checked in polynomial time whether a given assignment is certainly $\mathrm{PO}$ or not (Corollary 1). To prove NP-hardness, we adapt the proof of Theorem 7.

The proof of Theorem 7 involved a reduction from SERIALDiCTATORSHIPFEASIBILITY to the problem ExistsCERTAINLYPO-AssignMENT for the lottery preference model. In particular, it was shown that there is a yes instance of SERIalDictatorshipFEasibility if and only if there exists a certainly PO assignment for the constructed lottery preferences.

Recall that in the proof of Theorem 7 the lottery preferences are constructed as follows in the reduction. The set of agents and items is the same as in the original problem SERIALDictatorshipFEAsibiLity. Each agent has two possible preferences each with probability 0.5 . Each agent $j \in N$ now has two preference lists where one of them is $\succ_{j}$. For agent $i$, we add another preference list $\succ_{i}^{\prime}$ in which $i$ 's most preferred item is $o$ and the rest of the items are in the same order as in $\succ_{i}$. For each other agent $j \in N \backslash\{i\}$, we add a preference list $\succ_{j}^{\prime}$ which is identical to $\succ_{j}$ except that $o$ is moved to the end of the list.

We now show that the particular lottery preferences involved in the reduction have a one-to-one correspondence with uncertain preferences in the ranking model. The uncertainty lottery preferences of each agent $k$ induce a corresponding ranking matrix $M_{k}$ that consists of $1 \mathrm{~s}, 0 \mathrm{~s}$ and $1 / 2 \mathrm{~s}$ entries. The matrices are specified as follows.

Let $r_{i}(o)$ be the rank of item $o$ agent $i$ in preference $\succ_{i}$. If $r_{i}(o) \neq 1$, then $M_{i}(o, 1)=0.5$ and $M_{i}\left(o, r_{i}(o)\right)=0.5$. If $r_{i}(o)=1$, then $M_{i}(o, 1)=1$. The other entries of the row $M_{i}(o)$ are all zeroes. Now consider some item $o^{\prime} \neq o$. If $o \succ_{i} o^{\prime}$, then $M_{i}\left(o^{\prime}, r_{i}\left(o^{\prime}\right)\right)=1$. If $o^{\prime} \succ_{i} o$, then $M_{i}\left(o^{\prime}, r_{i}\left(o^{\prime}\right)\right)=0.5$ and $M_{i}\left(o^{\prime}, r_{i}\left(o^{\prime}\right)+1\right)=0.5$. The other entries of row $M_{i}\left(o^{\prime}\right)$ are all zeroes.

If $r_{j}(o)=n$, then $M_{j}(o, n)=1$. If $r_{j}(o)<n$, then $M_{j}\left(o, r_{j}(o)\right)=0.5$ and $M_{j}(o, n)=0.5$. The other entries of row $M_{j}(o)$ are all zeroes. Now consider some item $o^{\prime} \neq o$. If $o^{\prime} \succ_{j} o$, then $M_{i}\left(o^{\prime}, r_{j}\left(o^{\prime}\right)\right)=1$. If $o \succ_{j} o^{\prime}$, then $M_{j}\left(o^{\prime}, r_{i}\left(o^{\prime}\right)\right)=0.5$ and $M_{j}\left(o^{\prime}, r_{i}\left(o^{\prime}\right)-1\right)=0.5$. The other entries of the row $M_{j}\left(o^{\prime}\right)$ are all zeroes.

We now claim that the lottery model preferences constructed are the unique lottery preferences consistent with agents' ranking uncertain preferences. Consider any decomposition of the matrix $M_{k}$ into permutation matrices. Each permutation matrix can be viewed as encoding an injection from the set of items to their ranking for agent $k$. If $k=i$, then for agent $i$, in one of the permutation matrices $P_{i}^{2}, i$ has to have $o$ in the first position, and in another 
permutation matrix $P_{i}^{1}, i$ has $o$ in the same ranking as in the preference $\succ_{i}$. In $P_{i}^{2}$, when $i$ has $o$ in the first position, $i$ has her original first ranked item in the second position, her original second ranked item in the third position, and so on until we reach o's original ranked position. Hence $P_{i}^{2}$ corresponds to preference $\succ_{i}^{\prime}$. By a similar argument $P_{i}^{1}$ corresponds to preference $\succ_{i}$. If $k=j \neq i$, then by a similar argument, one of the permutation matrices $P_{j}^{1}$ corresponds to $\succ_{j}$ and the other permutation matrix $P_{j}^{2}$ corresponds to $\succ_{j}^{\prime}$.

It is clear that the uncertain lottery preferences $\succ_{k}$ and $\succ_{k}^{\prime}$ with probability half each resulted in uncertain ranking matrix $M_{k}$. We also showed that $M_{k}$ can only be achieved by the unique probability distribution of half probability each over deterministic preferences $\succ_{k}$ and $\succ_{k}^{\prime}$. Thus, we have established that the ranking preference represented by $M_{k}$ are equivalent to the lottery preferences. Therefore there exists a certainly PO assignment in the ranking preferences model if there exists a certainly PO assignment in the other lottery preference model. Hence, it follows that there exists a certainly PO assignment for the ranking model if and only if we have a yes instance of SERIALDictaTORSHIPFEASIBILITY.

Theorem 17 gives us the following corollary.

Corollary 9. For the ranking model, AssignmentWithHighestPO-Prob is NP-hard.

Next we show that PO-ProbabILITY is \#P-complete for instances for which PO probability is well defined because there is a unique and compact lottery model representation for the ranking model preferences. Hardness for \#P can be shown by using the reduction used in the proof of Theorem 12 .

Theorem 18. For the ranking model, PO-Probability is \#P-complete.

Proof. It is straightforward to show that PO-ProbabiLITy is in \#P. Hardness for \# $\mathrm{P}$ can be shown by using the reduction used in the proof of Theorem 12 . In this reduction, each agents either has a certain linear preference, or a preference where they are indifferent between their two most preferred items (and have a certain linear preference over all other items). These preferences can straightforwardly be expressed in the ranking model. Thus, for the ranking model, PO-Probability is \#P-hard as well.

\section{Conclusions}

We presented some general characterization and algorithmic results that apply to large classes of uncertainty models. We then extended the study to five uncertainty models, three of which are especially compact (at most polynomial in the number of agents and items). We see that as we move from deterministic preferences to uncertain preferences, the complexity of computing Pareto optimal outcomes jumps significantly even though the input for problems we study may not be compact. The computational hardness results carry over to 
more complex models in which there may be more items than agents, agents may have capacities, and items may have copies. In the paper, we made an assumption that the underlying preferences are strict. It will be interesting to explore the situation when the underling preferences could be weak. A deeper parametrized complexity analysis of the problems is a promising research direction. An orthogonal but equally interesting direction will be to consider other fairness, stability, or efficiency desiderata.

\section{Acknowledgments}

This paper includes results from two conference papers $[8,4]$. We are grateful to the anonymous IJCAI and AAAI reviewers for their helpful comments. Haris Aziz is supported by a Scientia Fellowship. Péter Biró acknowledges the support of the Hungarian Academy of Sciences under its Momentum Programme (LP2016-3/2018) and Cooperation of Excellences Grant (KEP-6/2018), and the Hungarian Scientific Research Fund, OTKA, Grant No. K128611. Ronald de Haan is supported by the Austrian Science Fund (FWF), project J4047.

[1] Abdulkadiroğlu, A., Sönmez, T., 1998. Random serial dictatorship and the core from random endowments in house allocation problems. Econometrica $66(3), 689-701$.

[2] Abdulkadiroğlu, A., Sönmez, T., 1999. House allocation with existing tenants. Journal of Economic Theory 88, 233-260.

[3] Abraham, D., Cechlárová, K., Manlove, D., Mehlhorn, K., 2004. Pareto optimality in house allocation problems. In: Proceedings of the 15th Annual International Symposium on Algorithms and Computation (ISAAC). Vol. 3341 of Lecture Notes in Computer Science. Springer, pp. 3-15.

[4] Aziz, H., Biró, P., de Haan, R., Rastegari, B., 2019. Pareto optimal allocation under compact uncertain preferences. In: Proceedings of the 33rd Proceedings of the AAAI Conference on Artificial Intelligence.

[5] Aziz, H., Biró, P., Fleiner, T., Gaspers, S., de Haan, R., Mattei, N., Rastegari, B., 2017. Stable matching with uncertain pairwise preferences. In: Proceedings of the 16th Proceedings of the International Joint Conference on Autonomous Agents and Multiagent Systems (AAMAS). pp. 344-352.

[6] Aziz, H., Biró, P., Gaspers, S., de Haan, R., Mattei, N., Rastegari, B., 2016. Stable matching with uncertain linear preferences. In: Proceedings of the 9th International Symposium on Algorithmic Game Theory (SAGT). pp. $195-206$.

[7] Aziz, H., Biró, P., Lang, J., Lesca, J., Monnot, J., 2016. Optimal reallocation under additive and ordinal preferences. In: Proceedings of the 15 th International Conference on Autonomous Agents and Multi-Agent Systems (AAMAS). pp. 402-410. 
[8] Aziz, H., de Haan, R., Rastegari, B., 2017. Pareto optimal allocation under uncertain preferences. In: Proceedings of the 26th International Joint Conference on Artificial Intelligence (IJCAI). pp. 1472-1474.

[9] Aziz, H., de Keijzer, B., 2012. Housing markets with indifferences: a tale of two mechanisms. In: Proceedings of the 26th Proceedings of the AAAI Conference on Artificial Intelligence. pp. 1249-1255.

[10] Aziz, H., Lang, J., Monnot, J., 2016. Computing Pareto optimal committees. In: Proceedings of the 25th International Joint Conference on Artificial Intelligence (IJCAI). pp. 60-66.

[11] Bogomolnaia, A., Moulin, H., 2001. A new solution to the random assignment problem. Journal of Economic Theory 100 (2), 295 - 328.

[12] Brams, S. J., King, D. L., 2005. Efficient fair division: Help the worst off or avoid envy? Rationality and Society 17 (4), 387-421.

[13] Chevaleyre, Y., Endriss, U., Maudet, N., 2017. Distributed fair allocation of indivisible goods. Artificial Intelligence 242, $1-22$.

[14] Dopazo, E., Martínez-Céspedes, M. L., Jul. 2017. Rank aggregation methods dealing with ordinal uncertain preferences. Expert Systems with Applications 78 (C), 103-109.

[15] Drummond, J., Boutilier, C., 2014. Preference elicitation and interview minimization in stable matchings. In: Proceedings of the 28th AAAI Conference on Artificial Intelligence. pp. 645-653.

[16] Erdil, A., Ergin, H., 2017. Two-sided matching with indifferences. Journal of Economic Theory 171, 268-292.

[17] Flum, G., Grohe, M., 2004. The parameterized complexity of counting problems. SIAM Journal on Computing 33 (4), 892-922.

[18] Flum, J., Grohe, M., 2006. Parameterized Complexity Theory (Texts in Theoretical Computer Science). Springer-Verlag.

[19] Gärdenfors, P., 1973. Assignment problem based on ordinal preferences. Management Science 20, 331-340.

[20] Hazon, N., Aumann, Y., Kraus, S., Wooldridge, M., 2012. On the evaluation of election outcomes under uncertainty. Artificial Intelligence 189, $1-18$.

[21] Krysta, P., Manlove, D., Rastegari, B., Zhang, J., 2014. Size versus truthfulness in the house allocation problem. In: Proceedings of the 15th ACM Conference on Economics and Computation (ACM-EC). ACM Press, pp. 453-470. 
[22] Lovász, L., Plummer, M. D., 2009. Matching Theory. AMS Chelsea Publishing.

[23] Manlove, D., 2013. Algorithmics of Matching Under Preferences. World Scientific Publishing Company.

[24] Mazurek, J., 2017. Fuzzy rankings: Properties and applications. CoRR abs/1703.05201.

[25] Rastegari, B., Condon, A., Immorlica, N., Irving, R., Leyton-Brown, K., 2014. Reasoning about optimal stable matchings under partial information. In: Proceedings of the 15th ACM Conference on Economics and Computation. EC '14. ACM, New York, NY, USA, pp. 431-448.

[26] Rastegari, B., Condon, A., Immorlica, N., Leyton-Brown, K., 2013. Twosided matching with partial information. In: Proceedings of the 14th ACM Conference on Electronic Commerce. EC '13. ACM, New York, NY, USA, pp. $733-750$.

[27] Saban, D., Sethuraman, J., 2013. House allocation with indifferences: a generalization and a unified view. In: Proceedings of the 14th ACM Conference on Electronic Commerce (ACM-EC). ACM Press, pp. 803-820.

[28] Saban, D., Sethuraman, J., 2015. The complexity of computing the random priority allocation matrix. Mathematics of Operations Research 40 (4), 1005-1014.

[29] Svensson, L.-G., 1994. Queue allocation of indivisible goods. Social Choice and Welfare 11, 323-330.

[30] Svensson, L.-G., 1999. Strategy-proof allocation of indivisible goods. Social Choice and Welfare 16, 557-567.

[31] Valiant, L. G., 1979. The complexity of enumeration and reliability problems. SIAM Journal on Computing 8 (3), 410-421. 